\title{
Vortex configurations and critical parameters in superconducting thin films containing antidot arrays: Nonlinear Ginzburg-Landau theory
}

\author{
G. R. Berdiyorov,* M. V. Milošević, and F. M. Peeters ${ }^{\dagger}$ \\ Departement Fysica, Universiteit Antwerpen, Groenenborgerlaan 171, B-2020 Antwerpen, Belgium \\ (Received 14 July 2006; revised manuscript received 29 September 2006; published 16 November 2006)
}

Using the nonlinear Ginzburg-Landau (GL) theory, we obtain the possible vortex configurations in superconducting thin films containing a square lattice of antidots. The equilibrium structural phase diagram is constructed which gives the different ground-state vortex configurations as function of the size and periodicity of the antidots for a given effective GL parameter $\kappa^{*}$. Giant-vortex states, combination of giant- and multivortex states, as well as symmetry imposed vortex-antivortex states are found to be the ground state for particular geometrical parameters of the sample. The antidot occupation number $n_{o}$ is calculated as a function of related parameters and comparison with existing expressions for the saturation number $n_{s}$ and with experimental results is given. For a small radius of antidots a triangular vortex lattice is obtained, where some of the vortices are pinned by the antidots and some of them are located between them. Transition between the square pinned and triangular vortex lattices is given for different values of the applied field. The enhanced critical current at integer and rational matching fields is found, where the level of enhancement at given magnetic field directly depends on the vortex-occupation number of the antidots. For certain parameters of the antidot lattice and/or temperature the critical current is found to be larger for higher magnetic fields. Superconducting/normal $H-T$ phase boundary exhibits different regimes as antidots are made larger, and we transit from a plain superconducting film to a thin-wire superconducting network. Presented results are in good agreement with available experiments and suggest possible new experiments.

DOI: 10.1103/PhysRevB.74.174512 PACS number(s): 74.20.De, 74.25.Dw, 74.78.Na, 74.25.Ha

\section{INTRODUCTION}

Superconducting (SC) samples with periodic arrays of pinning sites have received much attention over the last decade. It is now well established that these artificial pinning centers (i) hold great potential for enhancing the critical parameters of the sample and (ii) give rise to different kinds of vortex behavior that is not observed in the presence of random pinning. In this respect, arrays of microholes (antidots) $)^{1-12}$ and submicron magnetic dots, ${ }^{13-15}$ have been studied, as their presence in the SC film strongly modifies the vortex structure compared to the one in nonpatterned films. ${ }^{16,17}$

Direct imaging experiments, ${ }^{1}$ magnetization and transport measurements, ${ }^{2-5}$ and theoretical simulations ${ }^{18-22}$ of vortex structures in samples with periodic pinning centers have shown that the vortices form highly ordered configurations at integer $H_{n}=n \Phi_{0} / S$ and at some fractional $H_{p / q}={ }_{q}{ }_{q} \Phi_{0} / S(n, p$, $q$ being integers) matching fields, where $\Phi_{0}=h c / 2 e=2.07$ $\times 10^{-7} \mathrm{G} \mathrm{cm}^{2}$ is the flux quantum, and $S$ is the area of the primitive cell of the artificial lattice. This remarkable variety of stabilized vortex lattices may even be broadened by multiple possible degeneracies. These commensurability effects between the pinning array and the vortex lattice are responsible for an enhanced pinning and consequently increased critical currents. Very recently Karapetrov et al. ${ }^{23}$ investigated vortex configurations in a single crystal superconducting heterostructure with an array of submicron normal metal islands by scanning tunneling microscopy. They observed the coexistence of strongly interacting multiquanta vortex lattice with interstitial Abrikosov vortices. Different vortex phase transitions are given, which occur when the number of magnetic flux quanta in the sample changes.
Motivated by those experimental studies on perforated superconductors, significant efforts have been made on the theoretical side as well. For example, extensive molecular dynamics simulations ${ }^{18-22}$ in the London limit have been performed in an attempt to calculate the vortex structure and their dynamics in a periodic pinning potential. Although the general behavior of vortex lattices was accurately described, made approximations are valid only in certain range of parameters. Namely, in the London approach, vortices are considered as classical point-particles (with different models for their interaction) and the influence of the antidots is introduced through model hole potential, which in principle can never be generalized. Recently, Nordborg and Vinokur ${ }^{24}$ discussed in the detail interaction of vortices with an arbitrarily large cavity, but still within the London theory. This study was actually an extension of the work of Mkrtchyan and Shmidt, ${ }^{25}$ who crudely estimated the maximum possible number of vortices trapped by a single insulating inclusion with radius $R$ as $n_{s} \cong R / 2 \xi(T)$, where $\xi(T)$ is the temperature dependent coherent length. For regular arrays of pinning centers the saturation number becomes $n_{s} \sim[R / \xi(0)]^{2}$ due to the vortex-vortex interactions. ${ }^{26}$ The antidot-vortex interaction and the following maximal occupation number of each antidot appear to be crucial for many phenomena. For example, experiments on thin films with a lattice of holes showed a "localization transition:" 27 all vortices drop inside the holes when the coherence length becomes larger than the interhole spacing. In Ref. 4 it was shown that the antidot size realizing the optimum pinning is actually field dependent. The effective vortex-pinning potential and saturation number of the pinning sites for different temperature and applied dc fields were recently investigated experimentally by means of ac susceptibility measurements, for superconducting films with 
an array of antidots ${ }^{7,8}$ and for the case of not fully perforated holes (i.e., blind holes). ${ }^{10}$

Most of the experiments on perforated superconducting films are carried out in the effective type-II limit $\left(\kappa^{*}\right.$ $=\lambda^{2} / d \xi \gg 1 / \sqrt{2}, d$ being the thickness of the superconducting film and $\lambda$ the magnetic penetration depth). In this regime, the vortices act like charged point particles and their interaction with periodic pinning potential can be described using molecular dynamic simulations. ${ }^{18-21}$ However, the overlap of vortex cores (with size $\sim \xi$ ), and the exact shape of the intervortex interaction (depending on the superconducting material properties reflected through $\kappa$ ), may significantly modify the vortex structures and consequently the critical current when this criteria is no longer satisfied.

Besides, the vortex-pinning and the critical current enhancement, higher critical field $\left(H_{c 3}\right)$ near an open circular hole in a thin film (the so-called "surface superconductivity") has been predicted theoretically ${ }^{28}$ and confirmed experimentally. ${ }^{29}$ Cusps in the $H-T$ boundary were observed, which occurs when the number of vortices which nucleate inside the hole increases by one, similarly to the known Little-Parks effect. The ratio between the critical fields in perforated samples was estimated in limiting cases: $H_{c 3} / H_{c 2}=1$ when $R \rightarrow 0$ (or $R \ll \xi$ ) and $H_{c 3} / H_{c 2}=1.695$ when $R \rightarrow \infty$.

In this work superconducting films with square arrays of antidots are treated within the phenomenological GinzburgLandau theory. This approach considers vortices as extended objects and no approximations must be made on, e.g., the vortex-vortex interaction and/or the vortex-antidot interaction. In Sec. II, the details of our numerical formalism are given. Section III deals with vortex lattices in perforated films in homogeneous magnetic field, with emphasis on the number of pinned and interstitial vortices as function of the antidot-size and interhole distance. In case of weak pinning potentials, i.e., small size anti-dots, we discuss the triangle to square vortex lattice transition in Sec. IV. In Sec. V, we address the behavior of critical current in the sample as a function of the applied field, for different geometrical parameters, and temperature. The dependence of the critical field on temperature, and different regimes in the $H-T$ phase diagram are discussed in Sec. VI for different antidot size. All presented findings are then summarized in Sec. VII.

\section{THEORETICAL FORMALISM}

In this work, we consider a thin superconducting film (of thickness $d \ll \xi, \lambda$ ) with a square array of holes (radius $R$, period $W$ ) immersed in an insulating media in the presence of a perpendicular uniform applied field $H$ (see Fig. 1). To describe the superconducting state of the sample we solve the coupled nonlinear GL equations, which are written in dimensionless units in the following form: ${ }^{30,31}$

$$
\begin{gathered}
(-i \vec{\nabla}-\vec{A})^{2} \Psi=\Psi\left(1-|\Psi|^{2}\right), \\
-\kappa^{*} \Delta \vec{A}=\frac{1}{2 i}\left(\Psi^{*} \vec{\nabla} \Psi-\Psi \vec{\nabla} \Psi^{*}\right)-|\Psi|^{2} \vec{A} .
\end{gathered}
$$

We measure the distance in units of the coherence length $\xi$, the vector potential $\vec{A}$ in $c \hbar / 2 e \xi$, the magnetic field $H$ in

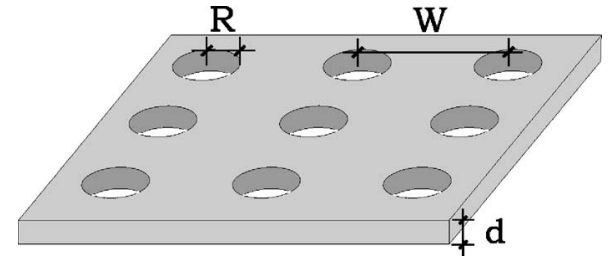

FIG. 1. Schematic view of the sample: a superconducting film (thickness $d$ ) with a regular array (period $W$ ) of circular antidots (radius $R$ ).

$H_{c 2}=c \hbar / 2 e \xi^{2}=\kappa \sqrt{2} H_{c}$, and the order parameter $\Psi$ in $\sqrt{-\alpha / \beta}$ with $\alpha, \beta$ being the GL coefficients.

The magnitude of the applied magnetic field $H=n \Phi_{0} / S$ is determined by the number $n$ of flux quanta $\Phi_{0}=h c / 2 e$ $=2.07 \times 10^{-7} \mathrm{G} \mathrm{cm}^{2}$ piercing through the rectangular simulation area $S=W_{x} \times W_{y}=N_{x} N_{y} W^{2}$, with $W_{x(y)}=N_{x(y)} W$ and $N_{x}$ and $N_{y}$ as integers. At the superconductor-insulator interface we impose the boundary condition corresponding to zero normal component of the superconducting current. The periodic boundary conditions for $\vec{A}$ and $\Psi$ (simulating the periodicity of both superconducting film and antidot lattice) have the form ${ }^{32}$

$$
\begin{gathered}
\vec{A}\left(\vec{\rho}+\vec{b}_{i}\right)=\vec{A}(\vec{\rho})+\vec{\nabla} \eta_{i}(\vec{\rho}), \\
\Psi\left(\vec{\rho}+\vec{b}_{i}\right)=\Psi \exp \left[2 \pi i \eta_{i}(\vec{\rho}) / \Phi_{0}\right],
\end{gathered}
$$

where $\vec{b}_{i}(i=x, y)$ are lattice vectors, and $\eta_{i}$ is the gauge potential. These boundary conditions imply that $\vec{A}, \Psi$ are invariant under lattice translations combined with specific gauge transformations $\eta_{x, y}$. Other quantities, such as the magnetic field, the current or the order parameter density are periodic. We use the Landau gauge $\vec{A}_{0}=H x \vec{e}_{y}$ for the external vector potential and $\eta_{x}=H W_{x} y+C_{x}, \eta_{y}=C_{y}$, with $C_{x}, C_{y}$ being constants. ${ }^{15}$ Without antidot lattice, when the film is invariant under infinitely small translations, the free energy does not depend on $C_{x}, C_{y}$. The vortex lattice is only shifted relative to the simulation region when $C_{x}$ and $C_{y}$ are varied. This is not the case for an antidot lattice, when the change of these parameters leads to a displacement of the vortex lattice relative to the holes, leading to a variation of the free energy. In general, one must minimize the free energy with respect to $C_{x}, C_{y}$. It can be shown that such a minimization gives a zero current when averaged over the cell area. We find that for a supercell having one hole, the optimal values are given by $C_{x, y}=0, \pm \pi$ and that for the supercell with $2^{N}$ holes the choice $C_{x, y}=0$ provides the minimum free energy.

We solved the system of Eqs. (1) and (2) self-consistently using the link variable approach ${ }^{33}$ in a finite-difference representation of the order parameter and the vector potential using a uniform Cartesian space grid $(x, y)$. The first GL equation is solved with a Gauss-Seidel iteration procedure. ${ }^{30}$ The vector potential is then obtained with the fast Fourier transform technique. The temperature is indirectly included in the calculation through the temperature dependence of the 


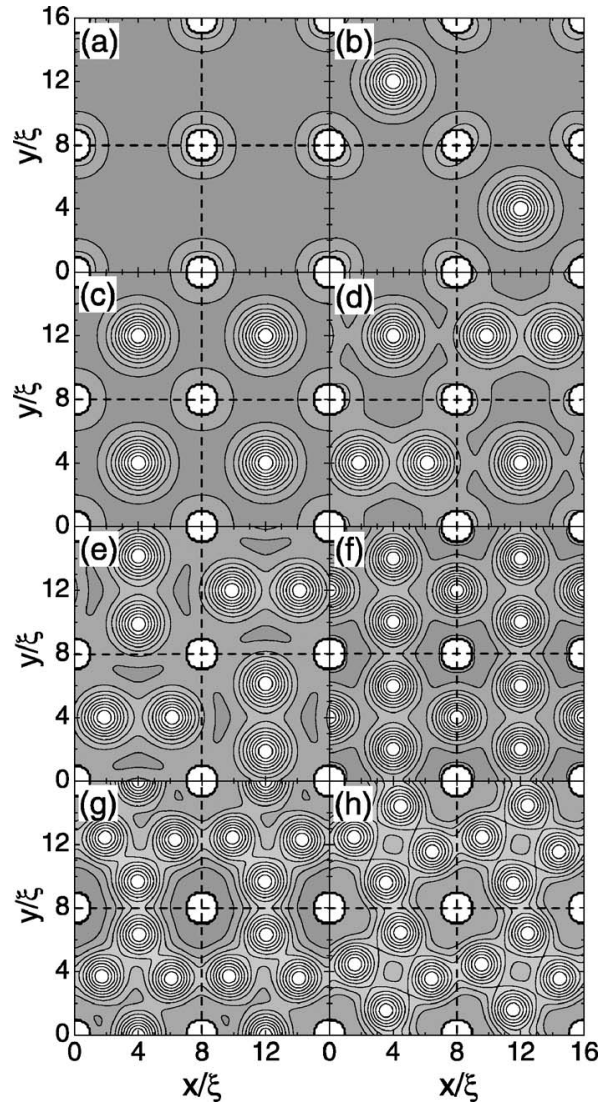

FIG. 2. Contour plots of the Cooper-pair density (white/dark color, low/high density) in the superconducting film with antidots of radius $R=\xi$ and interhole distance $W=8.0 \xi$ for the matching fields: $H_{n}=1$ (a), 3/2 (b), 2 (c), 5/2 (d), 3 (e), 4 (f), 9/2 (g), and 5 (h). The film thickness is $d=0.1 \xi$ and the effective GL parameter $\kappa^{*}=10$. Dashed lines indicate the antidot lattice.

coherence length $\xi(T)=\xi(0) / \sqrt{\left|1-T / T_{c 0}\right|}$ and penetration depth $\lambda(T)=\lambda(0) / \sqrt{\left|1-T / T_{c 0}\right|}$, where $T_{c 0}$ is the critical temperature at zero magnetic field.

\section{VORTEX LATTICES-INFLUENCE OF GEOMETRICAL PARAMETERS}

We first consider a supercell containing four holes $\left(W_{x}\right.$ $=W_{y}=2 W$ ) of radius $R$, with lattice period $W$ (see Fig. 1). Although our approach is valid for any integer number of flux-quanta piercing through the simulation region, we will restrict ourselves here to the so-called (integer and fractional) matching vorticities.

Figure 2 shows contour plots of $|\Psi|^{2}$ of the vortex lattice in case of antidots of radius $R=\xi$ and interhole distance $W$ $=8.0 \xi$ for different matching fields $H_{n}$. The film thickness is $d=0.1 \xi$ and the effective GL parameter $\kappa^{*}=\lambda^{2} / d \xi=10$. At the first matching field all vortices are trapped in the antidots [Fig. 2(a)]. Because of their small radius, each antidot is able to pin only one vortex, and additional vortices localize at interstitial sites when $H>H_{1}$ [see Fig. 2(b) for $H=H_{3 / 2}$ ]. At the second matching field [Fig. 2(c)] vortices occupy all interstitial sites, forming again a square lattice. For $H=H_{5 / 2}$,
[Fig. 2(d)] vortices form an ordered lattice with an additional vortex at every other interstice, added to the $H_{2}$ case. Note that the size of the vortex cores at neighboring interstitial sites differs: two vortices at the same interstice strongly interact and effectively bound each others core-areas, while the neighboring single interstitial vortex does not suffer from any lack of space resulting in its larger core. At the third matching field (three vortices per unit cell) two interstitial vortices in adjacent cells alternate in position [see Fig. 2(e)], preserving the twofold symmetry, but the vortex unit cell is 4 times the antidot lattice unit cell. At $H=H_{4}$ [Fig. 2(f)] we observe the first evidence of the competition between the Abrikosov vortex lattice (characteristic for thin film superconductors) and the symmetry of the pinning lattice, as vortices (including pinned ones) form a slightly deformed hexagonal lattice. Notice that for $H=H_{9 / 2}$ [Fig. 2(g)] the number of vortices per antidot unit cell is $n=9 / 2$ and the vortex lattice unit cell is twice the antidot unit cell. At the fifth matching field [Fig. 2(h)] the dense packing of vortices and their consequent strong interaction with the antidot lattice result in the restoration of the square lattice symmetry but the vortex lattice is tilted over $35^{\circ}$ with respect to the antidot lattice. Our results are in excellent agreement with the experiment of Ref. 1 and previous molecular dynamics simulations, ${ }^{19}$ in certain parameter range. Namely, the vortex configurations are mainly determined by the pinning force of each antidot, and the vortex-vortex interaction. The latter is very dependent on the density of vortex packing, as the known expressions for the vortex-vortex interaction do not take into account possible overlap of vortex cores. On the other hand, the antidot-vortex pinning potential is determined by the antidot-size and the period of the antidot lattice. Our approach takes all these aspects into account and their influence will be discussed in the remainder of this paper.

As shown in Fig. 2, for small hole radii, each antidot pins only one vortex (the hole occupation number $n_{o}$, the number of vortices sitting in the holes, equals 1), and the remaining vortices reside between the holes. One expects that, for larger hole radius $R$, vortex configurations with multiquanta vortices in each hole can become energetically preferable. ${ }^{23,28}$ Figure 3 shows the contour plots of the Cooper-pair density at the sixth matching field for different antidot radii. The antidot lattice period is $W=6 \xi$, the thickness is $d=0.1 \xi$ and $\kappa^{*}=10$. The number of vortices captured by each hole changes from one for $R=0.8 \xi$ [Fig. 3(a)] to five for $R=2.1 \xi$ [Fig. 3(e)]. The vortex arrangement outside the holes is determined not only by their mutual interaction, but also by the attraction with the antidots and the repulsion by their pinned vortices. For small radius $R$ a multivortex structure is found at the interstitial sites, as apparent from Figs. 3(a) and 3(b) (see also Fig. 2). By further increasing $R$ some of the vortices enter the holes and the remaining vortices are strongly caged between the antidots, resulting in the formation of giant vortices [Fig. 3(d)] and symmetry imposed vortex-antivortex pairs ${ }^{34}$ [Fig. 3(c)]. This is apparent from the contour plot of the phase of the order parameter $\Psi$ (righthand column of Fig. 3).

Similar behavior can be achieved if the hole size is kept the same, but the period of the hole lattice is decreased, as 

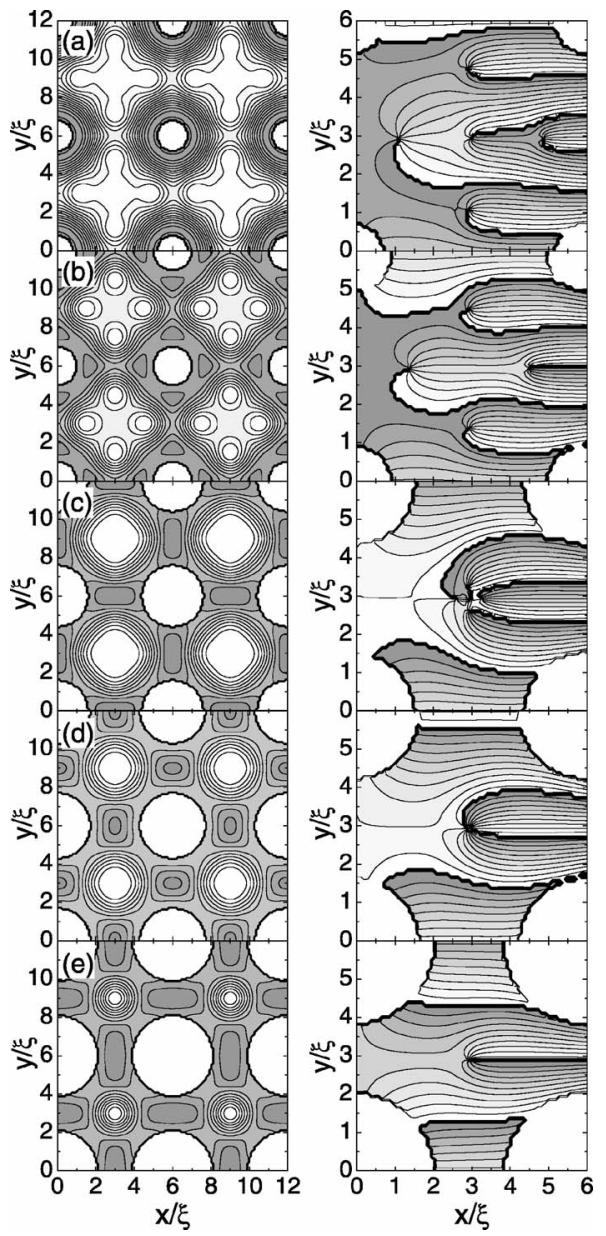

FIG. 3. Contour plots of the Cooper-pair density (left-hand column) and phase of the order parameter (right-hand column, limited to a single antidot lattice unit) for the sixth matching field $H=H_{6}$ and for different values of the hole radius: $R=0.8 \xi$ (a), $1 \xi(\mathrm{b}), 1.6 \xi$ (c), $1.7 \xi(\mathrm{d})$, and $2.1 \xi$ (e). The lattice period is $W=6 \xi$ and the GL parameter $\kappa^{*}=10$.

illustrated in Fig. 4. For small period $W$ the interstitial vortices form a giant vortices [Fig. 4(a)] because of the strong interaction with the pinned vortices in the antidots. With increasing $W$ one extra vortex is depinned and, due to the symmetry of the sample, vortex-antivortex pair with four vortices and one antivortex is formed in each interstitial site [Fig. 4(b)]. Further increase of $W$ leads to a triangular vortex structure at the interstitial sides with chosen orientation that minimizes the energy between neighboring cells [Figs. 4(c)-4(e)].

It is well known that the vortex-vortex interaction changes sign at the point $\kappa=1 / \sqrt{2}$. For $\kappa>1 / \sqrt{2}$, vortices repel each other while for $\kappa<1 / \sqrt{2}$ they attract. To see how this attractive interaction modifies the different vortex lattice configurations we consider a sample with small $\kappa$. Figure 5 shows the contour plots of the Cooper-pair density for $\kappa^{*}=10$ (type-II regime) and $\kappa^{*}=0.1$ (type-I regime) for the second, third, and fourth matching fields. For the given parameters of the sample and for $\kappa^{*}=10$ each hole pins one vortex and the remaining vortices sit at interstitial sites [Figs. 5(a)-5(c)]. The occupation number of each hole is increased to two in
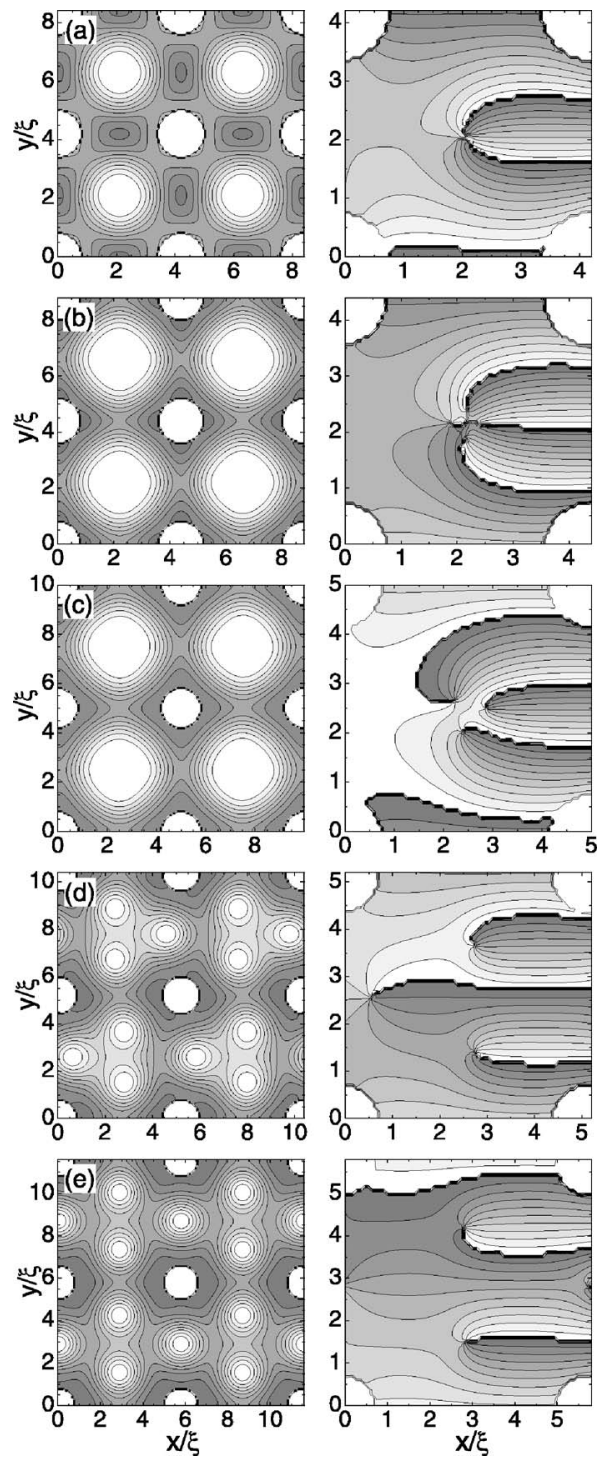

FIG. 4. Contour plots of the Cooper-pair density (left-hand column) and phase of the order parameter (right-hand column) for the fourth matching field $\mathrm{H}=\mathrm{H}_{4}$ and for the different values of the lattice period: $W=4.2 \xi$ (a), $4.4 \xi$ (b), $5 \xi$ (c), $5.2 \xi$ (d), and $5.8 \xi$ (e). The radius of the holes is $R=0.8 \xi$ and GL parameter $\kappa^{*}=10$.

the type-I sample [see Figs. 5(d)-5(i)] due to the enhanced expulsion of the magnetic field by the superconductor. Moreover, because of the attractive interaction between vortices, giant vortices become energetically more favorable contrary to the case for $\kappa^{*}=10$. Due to the instabilities of vortex states, which is common for type-I superconductors, variety of metastable vortex structures can be found (see also Ref. 35). As an example we show in Fig. 6 different metastable vortex states of the sample in Fig. 5 for $\kappa^{*}=0.1$. The free energies of those states are $F / F_{0}=-0.3268$ (a), -0.2823 (b), $-0.2787(\mathrm{c})$, and $-0.2755(\mathrm{~d})$. The ground state free energy [Figs. 5(f) and 5(i)] is $F / F_{0}=-0.3759$. Notice that because of the attractive interaction a giant vortex state is always favored.

To summarize the above findings, we constructed the equilibrium vortex phase diagram, which shows the depen- 


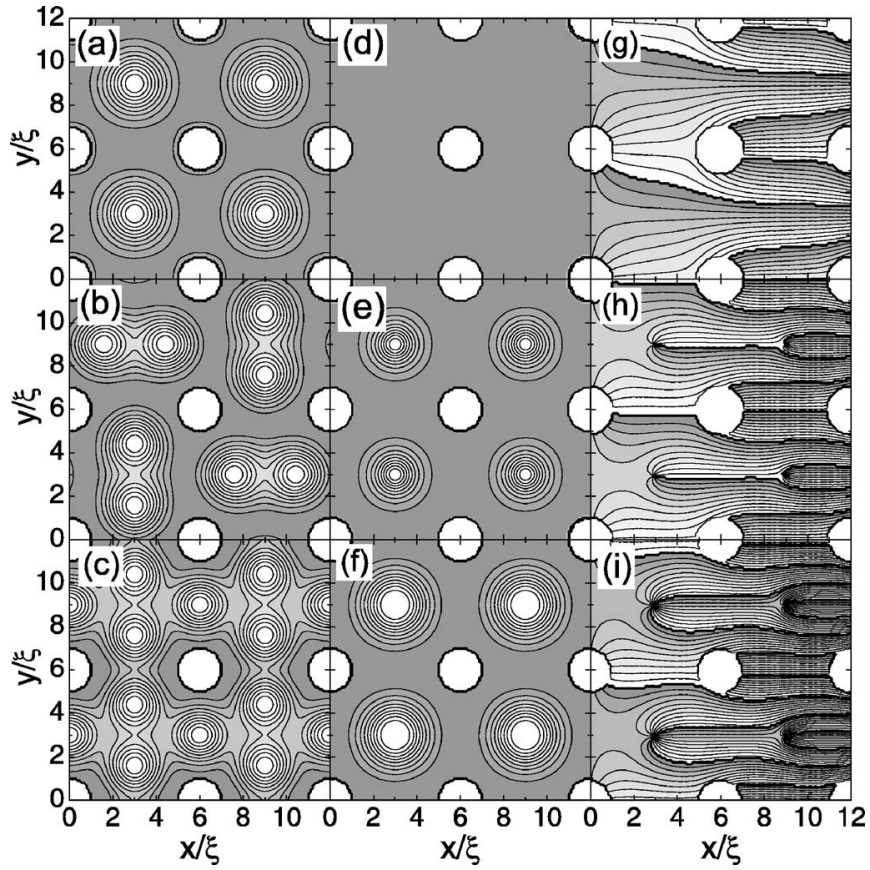

FIG. 5. Contour plots of the Cooper-pair density for $\kappa^{*}=10$ (a)-(c) and $\kappa^{*}=0.1$ (d)-(f). The lattice period is $W=6 \xi$ and the radius is $R=1 \xi$. Figures (g)-(i) show the phase of the order parameter of the states shown in (d)-(f). The first row is for $H=H_{2}$, the second for $H=H_{3}$, and the bottom row for $H=H_{4}$.

dence of antidot-occupation number $n_{o}$ on $R$ and $W$ for two values of $\kappa^{*}$, at the second, fourth, and sixth matching fields (Fig. 7). The ground- and metastable states are determined in our calculation by comparing the energy of all stable vortex states found when starting from different randomly generated initial conditions. The procedure of finding the free energy of the different metastable states was similar to that used for the case of mesoscopic superconducting disks. ${ }^{30,31}$ It should be noted, that an energetically unfavorable state remain stable in the wide range of variation of $R$ and $W$. Therefore, the transitions between the vortex states with different occupation numbers are of first order. It is seen from this figure that $n_{o}$ increases as the applied field is increased, which is in agreement with experimental results and theoretical predictions. ${ }^{2,12,23,28}$ With decreasing $\kappa^{*}$ the threshold hole radius for capturing another vortex decreases due to the smaller pinned vortex-interstitial vortex repulsion.

Let us compare our numerical results for the holeoccupation number $n_{o}$ with existing theoretical predictions. The saturation number $n_{s}\left(n_{s}=n_{o}\right.$ for larger fields) is usually estimated as $n_{s}=R / 2 \xi(T) .{ }^{25}$ Figure 8(a) shows the hole occupation number $n_{o}$ obtained from this expression and the one from our GL calculation for different period of the antidots. It is seen from this figure that this expression underestimates $n_{o}$ for small period $W$ (dashed and dotted curves). This is due to the fact that the last expression does not account for the interaction between vortices sitting at different holes. For larger period $W$ the occupation number is smaller in our calculation for a given radius of the holes (gray curve). A more accurate analysis was presented by Buzdin ${ }^{28}$ for bulk superconductors within the London approach. However, his

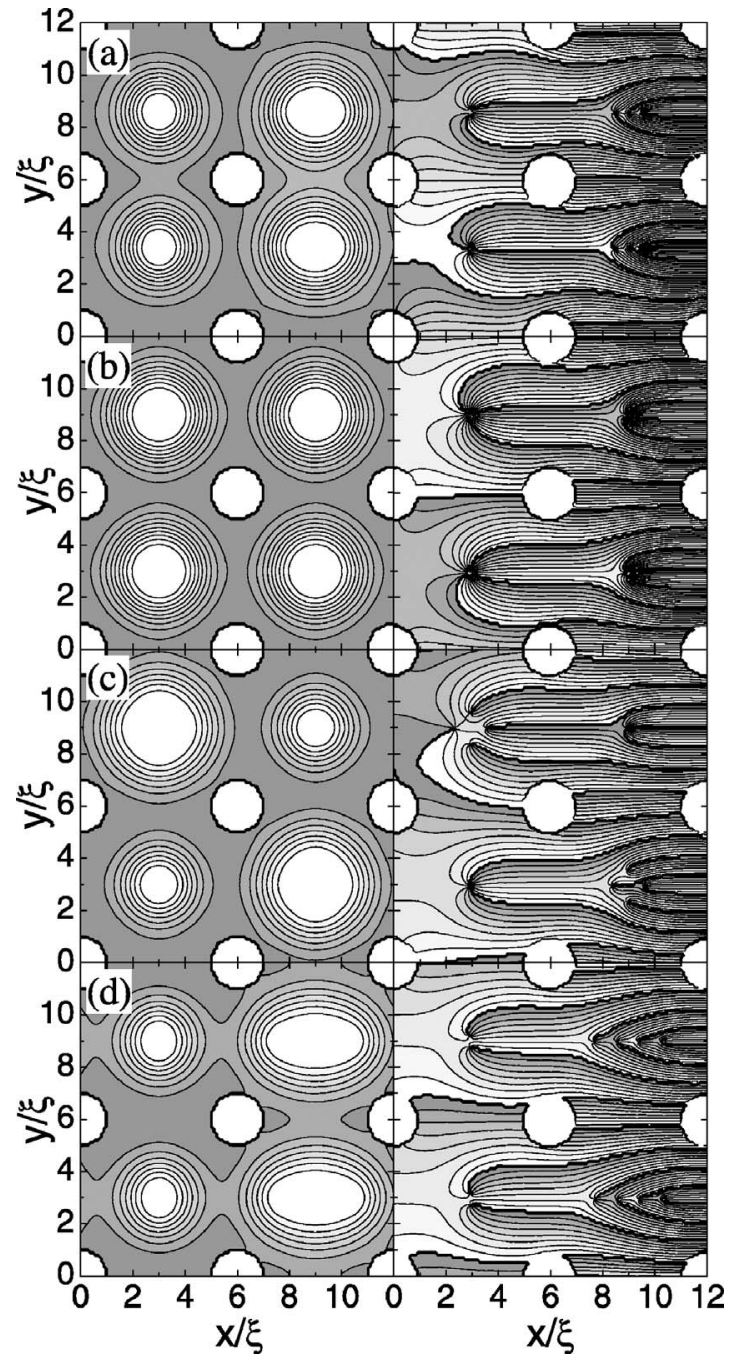

FIG. 6. Contour plots of the Cooper-pair density (left-hand column) and the phase of the order parameter (right-hand column) of the sample in Fig. 5 for different metastable vortex states at $H$ $=H_{4}$ and $\kappa^{*}=0.1$.

estimation of the critical hole radius $R / \xi \approx(W / \xi)^{2 / 3}$ (for $W$ $\ll \lambda$ ) and $R / \xi \approx \kappa^{2 / 3}$ (for $\lambda \ll W$ ) corresponding to the transition from single flux quantum to two flux quanta captured by the hole, differ from our numerically exact results, i.e., the magnitude of the critical hole radius is largely overestimated in Ref. 28 for both small and large period $W$ [see Fig. 8(b)].

The maximum number of flux quanta that can be trapped in a pinning center in a thin superconducting film was recently studied experimentally using scanning Hall probe microscopy ${ }^{11}$ and ac susceptibility measurements. ${ }^{8}$ In the latter case the saturation number was obtained from the transition to different dynamic regimes, as the interstitial vortices have higher mobility than those pinned by the antidots. They studied thin $\mathrm{Pb}$ films containing a square antidot array of period $d=1.5 \mu \mathrm{m}$. The antidots had circular (square) shape with radius $R=330 \mathrm{~nm}$ (size $a=0.8 \mu \mathrm{m}$ ), the film thickness was $d=80 \mathrm{~nm}(d=100 \mathrm{~nm})$ and the coherence length at zero temperature was estimated $\xi(0)=30 \mathrm{~nm}[\xi(0)=33 \mathrm{~nm}]$ in Ref. 11 (Ref. 8). Let us first discuss the results for the sample of Ref. 11, where the experimentally obtained saturation 


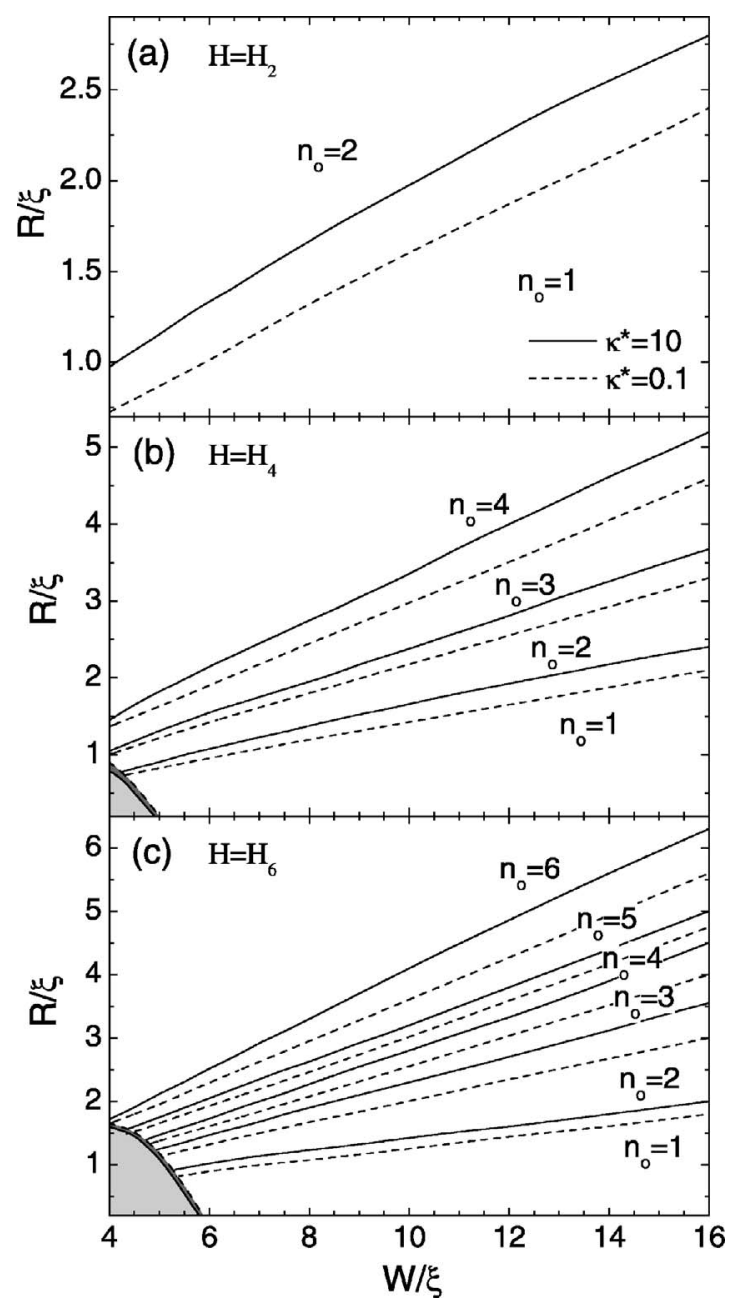

FIG. 7. The vortex phase diagram: the dependence of the hole occupation number $n_{o}$ on the radius of the holes $R$ and the distance between them $W$, for $\kappa^{*}=10$ (solid curves) and $\kappa^{*}=0.1$ (dashed curves) at the matching fields $H=H_{2}$ (a), $H=H_{4}$ (b), and $H=H_{6}$ (c). The gray region shows the normal state region for $\kappa^{*}=10\left(\kappa^{*}=0.1\right.$ when delimited by the full curve).

number was $n_{s}=2$ at $T / T_{c 0}=0.77$. Figure 9(a) shows the antidot occupation number $n_{o}$ as a function of temperature for different applied matching fields. At small applied fields $(H$ $\leqslant H_{3}$ ) the occupation number is equal to two, which is in agreement with the experimentally obtained $n_{s}$. With increasing applied field $\mathrm{H}>\mathrm{H}_{3}$ one more vortex is trapped by the holes, i.e., $n_{o}=3$, which is now larger than the experimental value. At higher temperatures $T>0.89 T_{c 0}, n_{o}$ again becomes equal to 2 . In this case one would estimate the saturation number from $n_{s} \approx R / 2 \xi(T)$ (Ref. 25) to be $n_{s}=1$ for $0.967 T_{c 0}<T<T_{c 0}$ and $n_{s}=2$ for $0.868 T_{c 0}<T<0.967 T_{c 0}$. We found the occupation number equal to $n_{o}=1$ only for the second matching field at the temperature range $0.985 T_{c 0}$ $<T<T_{c}$. The estimation of Buzdin ${ }^{28}$ for the critical hole radius $R^{3}<\xi(T) \lambda(T)^{2}$, where the transition from $n_{o}=1$ to $n_{o}=2$ occurs, gives the temperature range $T<0.985 T_{c 0}$. We found this transition at this temperature only for the second matching field. For larger fields the occupation number is always larger than unity. The giant vortex state is found only
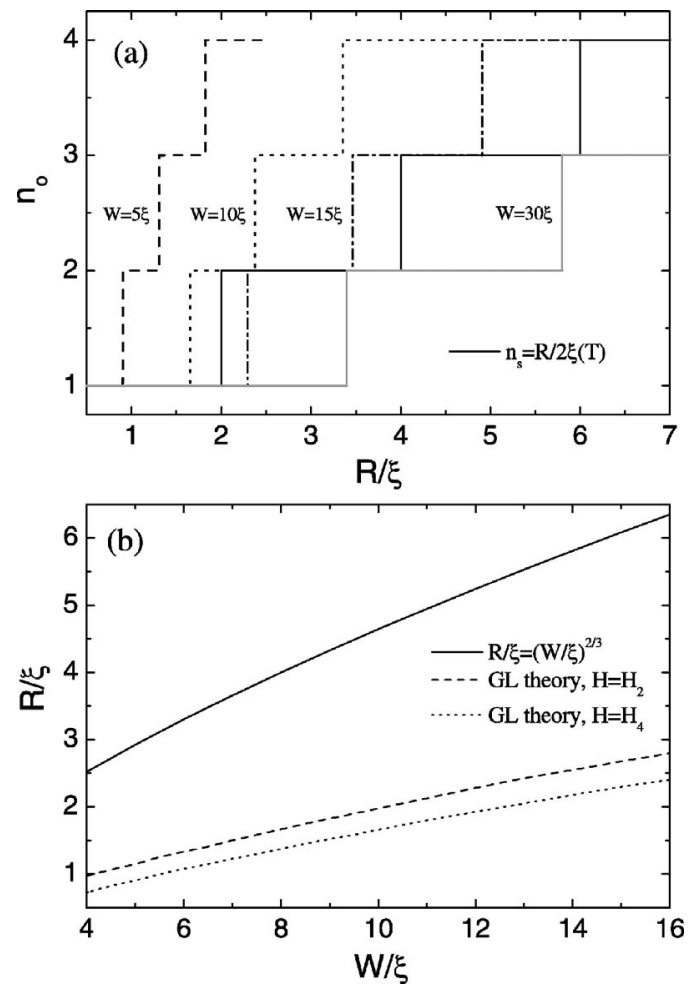

FIG. 8. (a) The antidot occupation number as a function of the antidot radius from the London theory (Ref. 25) $n_{s}=R / 2 \xi(T)$ (solid line) and from the GL theory for the period $W=5 \xi$ (dashed curve), $W=10 \xi$ (dotted curve), $W=15 \xi$ (dashed-dotted curve) and $W=30 \xi$ (gray curve) for the fourth matching field and $\kappa^{*}=10$. (b) The critical hole radius corresponding to $n_{o}=1 \rightarrow n_{o}=2$ transition as a function of the period $W$. Solid curve is obtained from the London theory (Ref. 28) and dashed (dotted) curve is the result from the GL theory for $H=H_{2}\left(H=H_{4}\right)$ and $\kappa^{*}=10$.

at $H=H_{4}$ for $T>0.984 T_{c 0}$ and the vortex-antivortex state is formed at $H=H_{5}$ for the temperatures $T>0.986 T_{c 0}$.

Up to now we use the temperature dependence for the coherence length and penetration depth as shown at the end of Sec. II which is obtained from the BCS theory ${ }^{36}$ and is valid near $T_{c}$. In this case the GL parameter $\kappa$ is temperature independent. In recent experiments on $\mathrm{Pb}$ arrays of nanowires arrays Stenuit et al. ${ }^{37}$ found that the following temperature dependence of the coherence length $\xi(T)$ $=\xi(0) \sqrt{\left|1-t^{4}\right|} /\left(1-t^{2}\right)$ and penetration depth $\lambda(T)$ $=\lambda(0) / \sqrt{1-t^{4}}$, which leads to a temperature dependence of the GL parameter $\kappa=\kappa(0) /\left(1+t^{2}\right)$, agrees better with experiment. Here $t=T / T_{c 0}$ and $\kappa(0)=\lambda(0) / \xi(0)$. These expressions are obtained from the two-fluid model. We calculated the hole occupation number $n_{o}$ using the above temperature dependencies, which is shown in the inset of Fig. 9(a). It is seen from this figure that the transition from $n_{o}=n$ to $n_{o}=n$ -1 occurs now at higher temperatures, but the results are qualitatively similar with the earlier results.

Figure 9(b) shows the occupation number $n_{o}$ as a function of temperature for different matching fields for the sample of Ref. 8. The experimentally obtained saturation number was $n_{s}=3$ for temperatures $T / T_{c 0}>0.97$. Our calculations give the same occupation number $n_{o}=3$ for this range of tempera- 


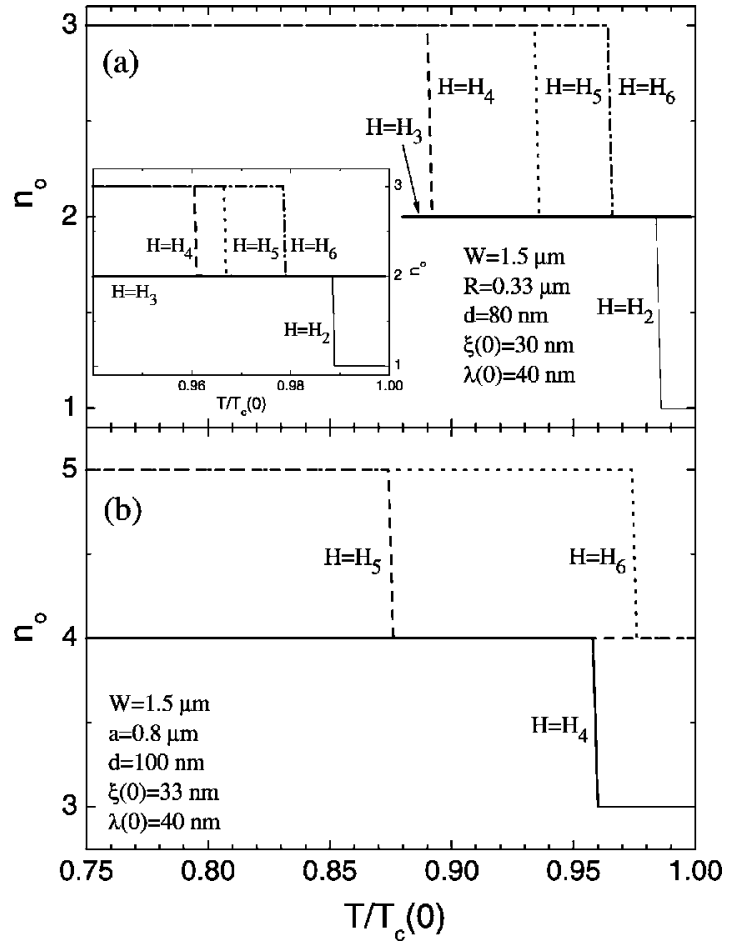

FIG. 9. The antidot occupation number $n_{o}$ as a function of temperature $T / T_{c 0}$ for different matching fields for circular antidots $(R=0.33 \mu \mathrm{m})$ (a) and square $(a=0.8 \mu \mathrm{m})$ (b) holes. The lattice period for both samples is $W=1.5 \mu \mathrm{m}$ and film thickness is $d$ $=80 \mathrm{~nm}$ (a) and $d=100 \mathrm{~nm}$ (b). The inset in (a) shows the hole occupation number $n_{o}$ of the same sample as a function of temperature for a different temperature dependence of $\xi(T)$ $=\xi(0) \sqrt{\left|1-t^{4}\right|} /\left(1-t^{2}\right)$ and $\kappa=\kappa(0) /\left(1+t^{2}\right)$, where $t=T / T_{c 0}$ and $\kappa(0)=\lambda(0) / \xi(0)$.

tures but only for fields $H \leqslant H_{4}$. The expression for the saturation number $n_{s} \approx a / 4 \xi(T)$ gives in this case $n_{s}=1$. At larger fields the occupation number increases, but still there will be interstitial vortices in the sample. ${ }^{23}$ These interstitial vortices lead to a larger dissipation in the sample which was used as the criterium for the determination of the saturation number $n_{s}$. But our calculations show that the appearance of interstitial vortices does not indicate the saturation of trapped vortices.

We have shown in our recent paper ${ }^{34}$ that a rich variety of ordered vortex structures: a combination of giant vortices with multivortices and vortex-antivortex pairs are found in perforated superconducting samples for fractional matching fields. Here we consider the dependence of these vortex states on the effective GL parameter $\kappa^{*}$. As an example we constructed the equilibrium vortex phase diagram for $H$ $=H_{9 / 2}$ rational matching field (i.e., 4.5 flux quanta per antidot) as a function of $R$ and $W$, and for $\kappa^{*}=10$ [Fig. 10(a)] and $\kappa^{*}=0.1$ [Fig. 10(b)]. In type-II regime [Fig. 10(a)], for larger period $W$ the vortex configuration always consists of individual vortices, except for $n_{o}$ flux lines pinned by each hole. One extra vortex per two holes is shared between the adjacent cells [insets (2), and (5)] or situated in every other cell [inset (7)]. With decreasing $W$, the interstitial vortices become strongly caged between the neighboring antidots, re-

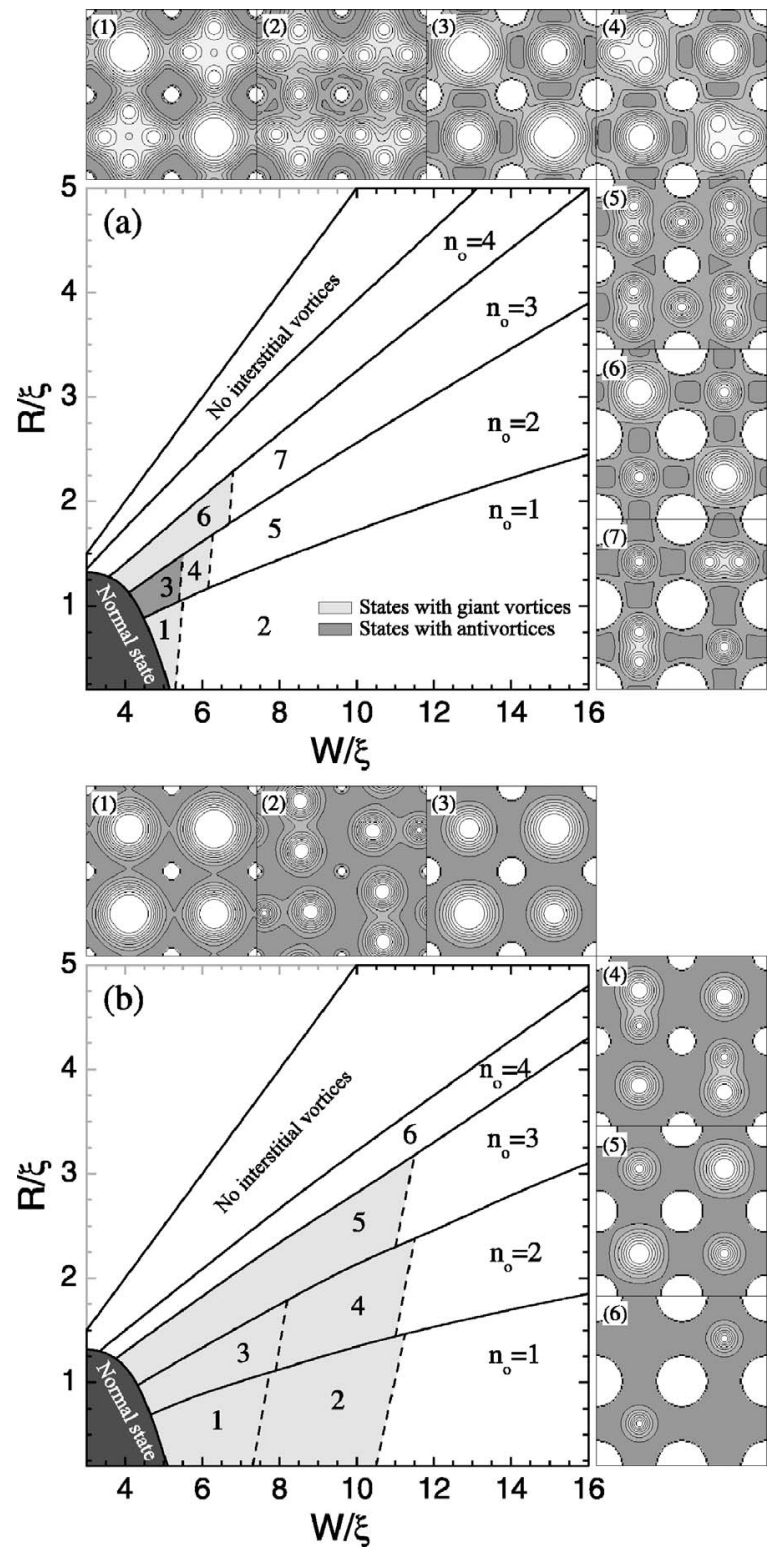

FIG. 10. The ground-state vortex lattice at $H=H_{9 / 2}$ as a function of the antidot-radius $R$ and their periodicity $W$, for $\kappa^{*}=10$ (a) and $\kappa^{*}=0.1$ (b). The solid lines denote the first order transitions between the states with different antidot-occupation number $n_{o}$, and dashed ones depict second order configurational transitions. The insets show the Cooper-pair density plots of the corresponding states indicated by the numbers in the phase diagram.

sulting in the distortion of the individual-vortex lattice. In this case, the $n$th matching field becomes larger than the second critical field for $W / \xi<\sqrt{2 \pi n}$. But the superconducting state in perforated films still survives due to enhanced superconductivity in close proximity around the holes (due to surface superconductivity). If the radius of the holes is then increased, individual vortices captured at interstitial sites can merge for $H>H_{c 2}$ to a giant vortex [region 1 in Fig. 10(a)]. This transition does not show any hysteretic behavior and is, therefore, of second order (similarly to the case for mesoscopic disks ${ }^{30}$ ). The creation of these giant vortex states is favored because of the repulsion of the vortices by the 
supercurrents around the holes (and consequent compression of vortices in the central part of the interstitial regions).

The influence of caging depends on the number of confined vortices; the combinations of giant vortices and multivortices may be formed in the interstitial sites [insets (1), (4), and (6)]. For interstitial vorticity 3 , a vortex-antivortex pair may nucleate, so that local vortex structure conforms with the square symmetry of the pinning lattice [see inset (3) in Fig. 10(a) and Ref. 34].

Figure 10(b) shows the ground-state phase diagram found for $\kappa^{*}=0.1$. Compared to Fig. 10(a), the threshold antidot radius for capturing another vortex decreases due to the enhanced screening of the applied field. Due to the attractive vortex-vortex interaction in type-I samples, giant-vortex states become energetically favorable at the interstitial sites and spread over the majority of the $W-R$ phase diagram (light gray areas). For a dense antidot lattice, giant vortices with different vorticity are found in adjacent cells $\{L=3$ and $L$ $=4$ [inset (1)], and $L=2$ and $L=3$ [inset (3)]\}. Contrary to the type-II case, these giant vortices can split to smaller giant vortices for larger spacing of antidots. They exhibit singlevortex behavior, forming the lattice of two quanta and single quantum vortices [insets (2) and (4)]. Such new quasiAbrikosov lattices of giant vortices result from the competition of vortex-vortex attraction and imposed square symmetry of pinning. At the same time, these competing interactions cause the complete disappearance of the vortexantivortex structures as found in type-II samples.

\section{TRIANGULAR TO SQUARE VORTEX LATTICE TRANSITION IN THE PRESENCE OF A SQUARE ANTIDOT LATTICE}

It is well known that the regular triangular vortex lattice has the lowest energy in superconductors with no pinning. ${ }^{17}$ As we have shown above the square lattice of pinning sites impose its own symmetry on the vortex structure. If the vortex-pinning strength in a periodic square array is reduced, the vortex-vortex repulsion starts to dominate over the pinning force and the triangular lattice is recovered. Transition between these phases was recently studied in Ref. 39 as a function of the amplitude of the vortex-pinning site interaction and the characteristic length scale of this interaction within the London theory (i.e., $\kappa^{*}=\infty$ ). They showed that the transition between triangular and square vortex lattice occurs for increasing strength of the pinning potential in the case of small values of pinning potential length scale. In Ref. 39 a model periodic pinning potential was introduced, the parameters of which are difficult to relate to any growth parameters of the sample.

To circumvent the latter problem we studied the case of weak pinning potential by introducing small antidots. Thus in order to decrease the pinning force in our calculations we just reduced the radius of the antidots $R$ for a given period of antidot lattice $W$. Calculations are done for a $8 \times 8$ unit cell $\left(W_{x}=W_{y}=8 W\right)$ with grid points $256 \times 256$. Figure 11 shows the phase diagram: the transition between the pinned (white region) and triangular (light gray region) vortex lattice as a function of the radius $R$ and period $W$ of antidots for the
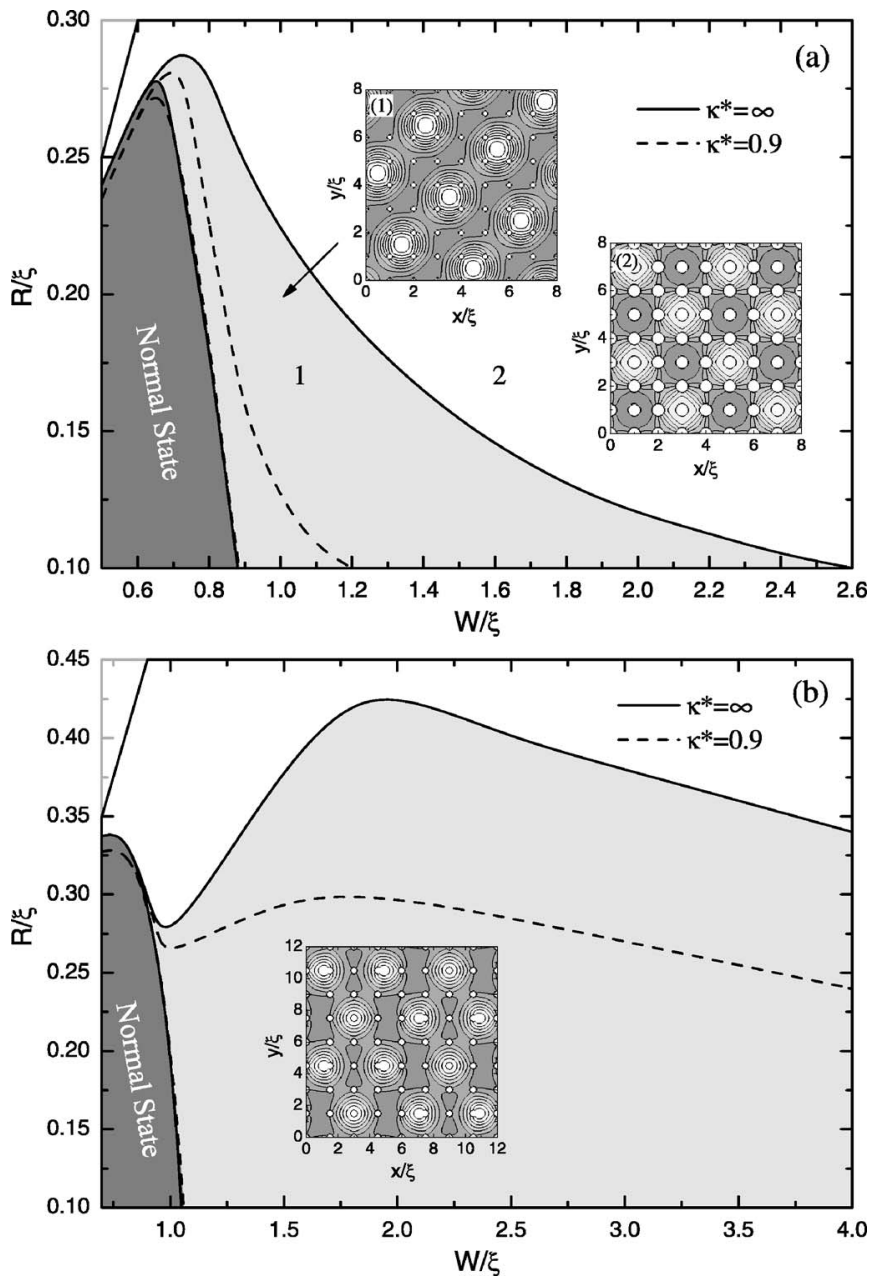

FIG. 11. The phase diagram: square (white region) and deformed triangular (light gray region) vortex lattice as a function of the radius $R$ and period $W$ of antidots at $H=H_{1 / 8}$ (a) and $H=H_{3 / 16}$ (b) for $\kappa^{*}=\infty$ (solid curves) and $\kappa^{*}=0.9$ (dashed curves). The insets show the Cooper-pair density plots of the corresponding states.

applied fields $H=H_{1 / 8}\left(\right.$ a) and $H=H_{3 / 16}$ (b). Let us first discuss the results for $H=H_{1 / 8}$. When the pinning strength is small (small $R$ ), it is energetically favorable to form a triangular lattice, where vortices are located between the holes [lefthand inset in Fig. 11(a)]. For larger radius of the holes a square vortex lattice becomes the ground state [right-hand side inset in Fig. 11(a)]. The critical radius of the holes $R$ to pin the vortices decreases with increasing period, contrary to the one corresponding to the case of two-flux quanta captured by the holes [see Fig. 8(b)]. If we decrease the GL parameter $\kappa$ the transition between pinned and triangular vortex lattices decreases (dashed curves in Fig. 11) due to the short range interaction between the vortices.

In Ref. 39 the phase diagram for the transition between the triangular and square vortex lattice was found to be the same for all submatching fields not exceeding $H=H_{1}$, except $H=H_{1}$ and $H=H_{1 / 2}$. Contrary to this results our calculations give a different phase diagram for different fractional matching fields. As an example, we show in Fig. 11(b) the transition lines between triangular and square pinned vortex configurations for $H=H_{3 / 16}$. The triangular vortex lattice is 


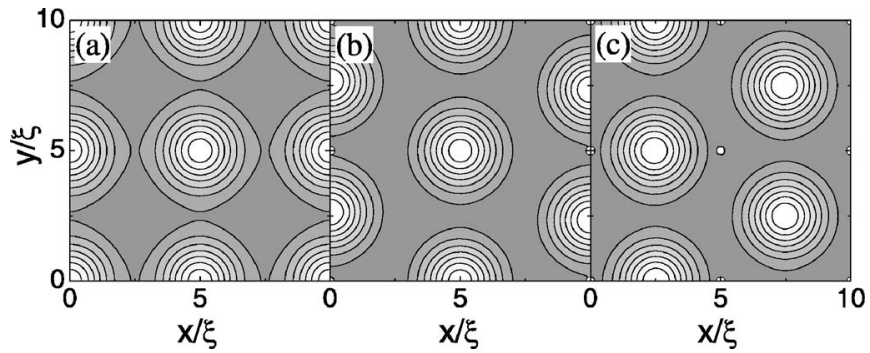

FIG. 12. Contour plots of the Cooper-pair density for different vortex states at $H=H_{1}$. The radius of the antidots is $R=0.14 \xi$, the period is $W=5 \xi$ and effective GL parameter is $\kappa^{*}=\infty$.

formed where some of the vortices are pinned and some of them are located between the antidots [see the inset of Fig. 11(b)]. For this value of the field the triangular vortex lattice is found for larger values of the period $W$ and radius $R$ of the antidots.

For the fields $H=H_{1}$ and $H=H_{1 / 2}$ the pinned vortex lattice has a square symmetry. An intermediate vortex configuration for these fields was obtained in Ref. 39 where vortices in odd rows of pinning centers are depinned and are located between the pinning sites forming a kind of triangular lattice. Our calculations show that such vortex configurations can be found only as a metastable state even for relatively larger values of the period $(4 \xi<W<6 \xi)$. To make this more clear we plot in Fig. 12 the ground state (a) and metastable (b), (c) vortex configurations at the first matching field. In addition to the triangular vortex state given in Ref. 39 [see Fig. 12(b)] another triangular vortex state is found, where all the vortices are located in the interstitial region [Fig. 12(c)].

\section{CRITICAL CURRENT OF PATTERNED SC FILMS}

In the preceding sections we showed that vortex configurations that are commensurate with the periodic arrays of antidots exhibits well-defined matching phenomena, which leads to pronounced peaks in the critical current (see, for example, Ref. 4). However, the stability of these vortex states strongly depend on the parameters of the sample. For example, a multiquanta vortex state become energetically favorable for large radius of the holes, while small holes can capture only a single vortex. The additional vortices located in interstitial sites reduces the critical current considerably. Therefore, we first investigate the critical current of our sample as a function of the relevant antidot parameters.

The first step to calculate the critical current is to accurately determine the vortex ground state for given applied magnetic field, in a manner described in the preceding section. Then the applied current in the $x$ direction is simulated by adding a constant $A_{c x}$ to the existing vector potential of the applied external field. ${ }^{15}$ With increasing $A_{c x}$ we find a critical value of $A_{c x}$ such that a stationary solution to Eqs. (1) and (2) cannot be found since a number of vortices is driven in motion by the Lorentz force. The current $j_{x}$ in the sample corresponding to the given value of $A_{c x}$ is obtained after integration of the $x$ component of the induced supercurrents in the $y$ cross section. The maximal achievable value of $j_{x}$ denotes the critical current $j_{c}$.

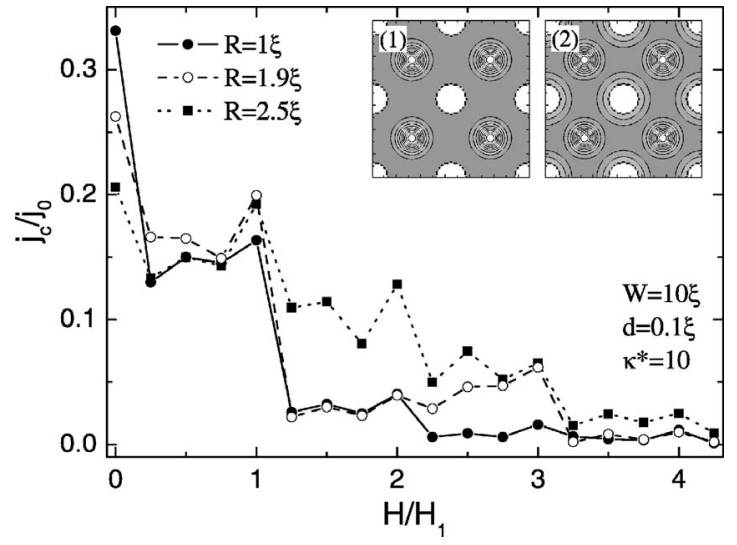

FIG. 13. Critical current density (in units of $j_{0}=c H_{c 2} \xi / 4 \pi \lambda^{2}$ ) as a function of the applied magnetic field (in units of the first matching field $H_{1}$ ) for three values of the antidot radius: $R=1 \xi$ (solid circles), $R=1.9 \xi$ (open circles), and $R=2.5 \xi$ (squares). The antidot lattice period is $W=10 \xi$, the film thickness is $d=0.1 \xi$ and the effective GL parameter is $\kappa^{*}=10$. The insets show the contour plots of the Cooper-pair density at the second (1) and third (2) matching fields for $R=1.9 \xi$.

Figure 13 shows the critical current density $j_{c}$ (in units of $\left.j_{0}=c H_{c 2} \xi / 4 \pi \lambda^{2}\right)$ as a function of applied magnetic field (normalized to the first matching field $H_{1}$ ) for different values of the antidot radius $R$ for fixed value of the antidot lattice period $W$. For small radius (solid circles), where only one vortex can be pinned by the hole, the peaks at the matching fields decrease with increasing applied field. The opposite behavior is found when there is a caging effect, ${ }^{40}$ i.e., $j_{c}\left(H_{n}\right)<j_{c}\left(H_{n+1}\right)$, which, e.g., is found for radius $R=1.9 \xi$ (open circles) for $n_{o}=1$ and $n_{o}=2$. This effect occurs when there are the same number of interstitial vortices but the number of pinned ones are different at the different matching fields. In this case the interstitial vortices feel a stronger repulsive interaction when there are a larger number of pinned vortices. As is shown in Fig. 13 (open circles), a higher critical current is found for the third matching field, when a double vortex occupies each hole and a single one is located at the interstitial, than for the second matching, with one vortex in each hole and a single interstitial vortex (see the insets of Fig. 13). This effect disappears with further increasing the radius $R$ due to the different occupation number $n_{o}$, i.e., no interstitial vortices at $\mathrm{H}=\mathrm{H}_{2}$.

In order to show the range of radius $R$ and period $W$ of antidots, where this caging effect is active, we constructed a $R$-W phase diagram for $H=H_{2}$ and $H=H_{3}$, shown in Fig. 14(a). The shadowed area indicates the vortex state with a single interstitial vortex for both $H=H_{2}$ (solid line) and $H$ $=H_{3}$ (dashed curves). Figure 14(b) shows the ratio $j_{c}\left(H_{3}\right) / j_{c}\left(H_{2}\right)$ as a function of period $W$. The critical radius $R$ is taken from the middle of the region (dotted curve). It is seen from this figure that, although we have the same vortex structure for all values of the period $4 \xi \leqslant W \leqslant 10 \xi$, the enhancement of $j_{c}$ is found only for $W \gtrsim 6.6 \xi$. For small period the pinned vortices at $\mathrm{H}=\mathrm{H}_{3}$ suppresses superconductivity around the holes and interstitial vortices are easily set into motion, reducing the critical current. 


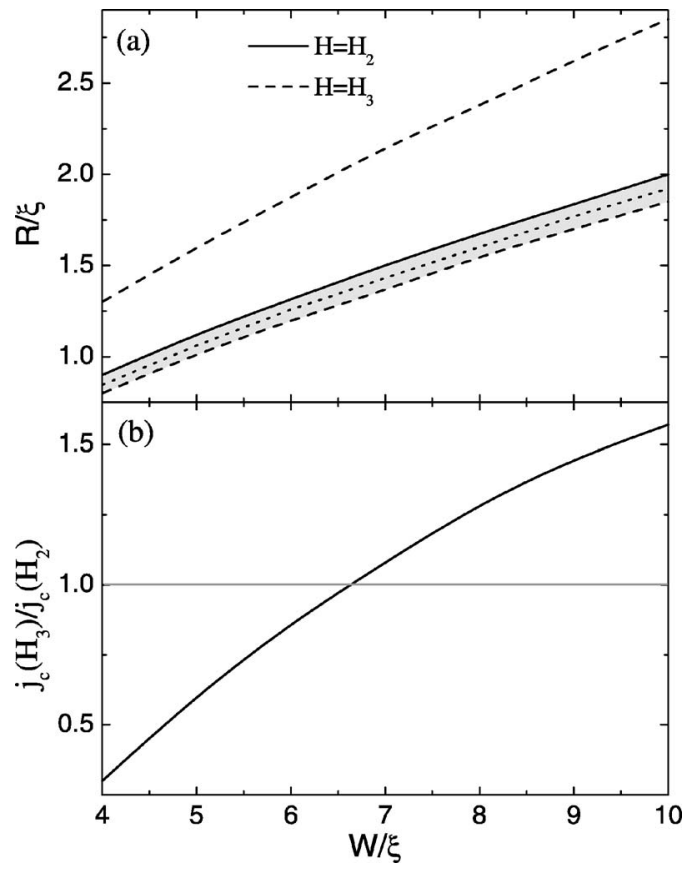

FIG. 14. (a) The dependence of the antidot occupation number $n_{o}$ as a function of antidot radius $R$ and lattice period $W$ (see also Fig. 7). The solid (dashed) line indicates the transition between the states with different $n_{o}$ at $H=H_{2}\left(H=H_{3}\right)$. The shadowed area indicates the vortex state with a single interstitial vortex for both applied fields (the occupation number in this region is $n_{o}=1$ for $H$ $=H_{2}$ and $n_{o}=2$ for $\left.H=H_{3}\right)$. (b) The ratio of $j_{c}\left(H_{3}\right) / j_{c}\left(H_{2}\right)$ as a function of the period $W$, where the radius of the antidots correspond to the dotted line in the middle of the dashed area.

Figure 15 shows the critical current density as a function of the field for two values of the period: $W=4 \xi$ (open circles) and $W=8 \xi$ (solid circles) at $R=1.25 \xi$. As we showed above, the $j_{c}(H)$ curve shows pronounced maxima at integer fields $H_{1}, H_{2}$, and $H_{3}$ and at some of the fractional matching fields. However, while the qualitative behavior of $j_{c}(H)$ in Fig. 15 is as expected, its quantitative behavior reveals a counterintuitive phenomenon. Namely, one expects higher critical current in the sample with larger interhole distance, simply due to the presence of more superconducting material. Indeed, that is the case for $H \leqslant H_{1}$, where the superconductor is able to compress all flux lines in the holes. However, for higher magnetic fields, the critical current drops sharply immediately after the first matching field $H_{1}$, which is related to the appearance of interstitial vortices. On the other hand, the smaller interhole distance affect the hole occupation number (see Fig. 7), and the additional vortices after $H=H_{1}$ are still captured by the holes (as illustrated by Cooper-pair density plots in the inset of Fig. 15). Consequently, the critical current in this case is larger for smaller periodicity. Note that even for smaller periodicity a sharp drop in $j_{c}$ is observed for $H>H_{1}$, as every additional vortex disturbs the stability of the vortex lattice. Even at $H=H_{2}$, although all vortices are captured by the holes, the critical current is lower, due to a stronger suppression of the order parameter around the holes compared to the $H=H_{1}$ case. The height of the matching peaks is decreasing with further increasing field (due to the

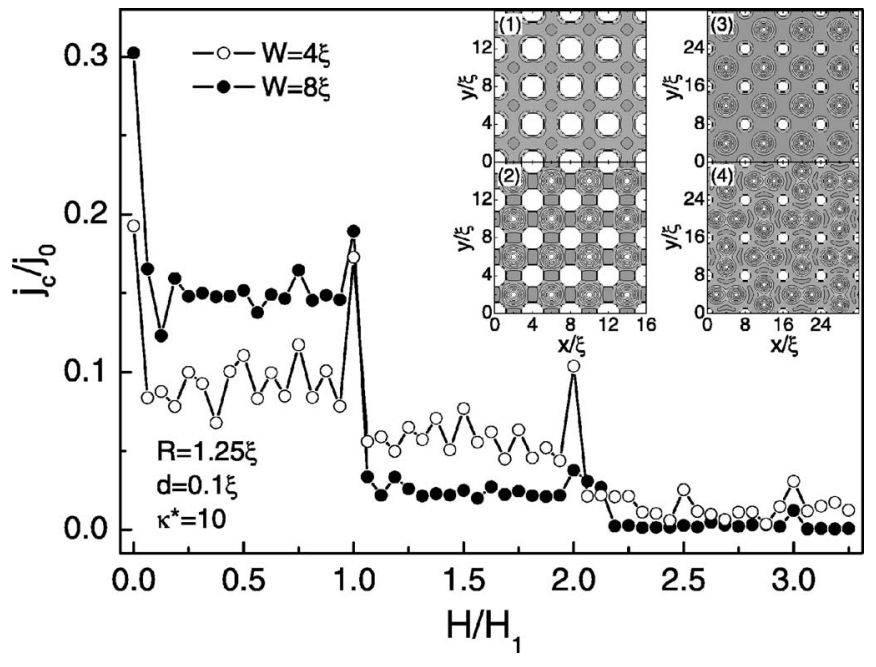

FIG. 15. Critical current density of the superconducting film as a function of the applied magnetic field for two values of the antidot lattice period: $W=8 \xi$ (solid circles) and $W=4 \xi$ (open circles). The insets show contour plots of the Cooper-pair density at the second $(1,3)$ and third $(2,4)$ matching fields for $W=4 \xi(1,2)$ and $W=8 \xi$ $(3,4)$. The radius of the holes is $R=1.25 \xi$, the film thickness is $d$ $=0.1 \xi$ and the effective GL parameter is $\kappa^{*}=10$.

presence of interstitial vortices), which agrees with experiment (see Ref. 4), and these peaks strongly diminish for higher fields as the vortex-flow overwhelms the pinning potential.

When we apply a dc current into the superconductor the vortex lattice is distorted before the vortices start moving. To illustrate this phenomenon, we plot in Fig. 16 the Cooper pair density of the superconducting film at the applied currents (in $y$ direction) $j=0$ (first column), $j=0.5 j_{c}$ (second column) $j=0.95 j_{c}$ (third column) for different matching fields. At the first [Fig. 16(a)] and second [Fig. 16(b)] matching fields all the vortices are displaced over the same distance, conserving the square symmetry in the lattice of vortices. At larger fields, when there is a large number of interstitial vortices [Figs. 16(c)-16(e)], the vortex configuration is changed by the current and some of the vortices are jammed at the interstitial sites. If we initially have giant vortices [Fig. 16(f)] they can be split into multivortices with increasing $j$. Our calculations also show that there is no transition from the multivortex state to the giant vortex state when we increase the applied current, and the occupation number of the antidots $n_{o}$ is found to be independent of $j$.

Another interesting feature following from the displacement is found for fractional matching fields. For example, Fig. 2(d) shows alternating two-vortex-single-vortex structure at $H=H_{5 / 2}$, where applying small current in the $y$ direction can shift the excess vortex from one interstitial site to another. Note that the resulting state has identical configuration and energy as the previous one. In order to estimate the energy barrier between these two vortex states we performed calculations for a superconducting film of thickness $d$ $=13 \mathrm{~nm}$ with an array of antidots with period $W=1 \mu \mathrm{m}$, radius $R=0.13 \mu \mathrm{m}$, at temperature $T=0.9 T_{c}$. We take $\xi(0)$ $=40 \mathrm{~nm}$ and $\lambda(0)=80 \mathrm{~nm}$, which are typical values for $\mathrm{Pb}$ thin films. We found an energy barrier of $\Delta F=6.2 \mathrm{meV}$, 


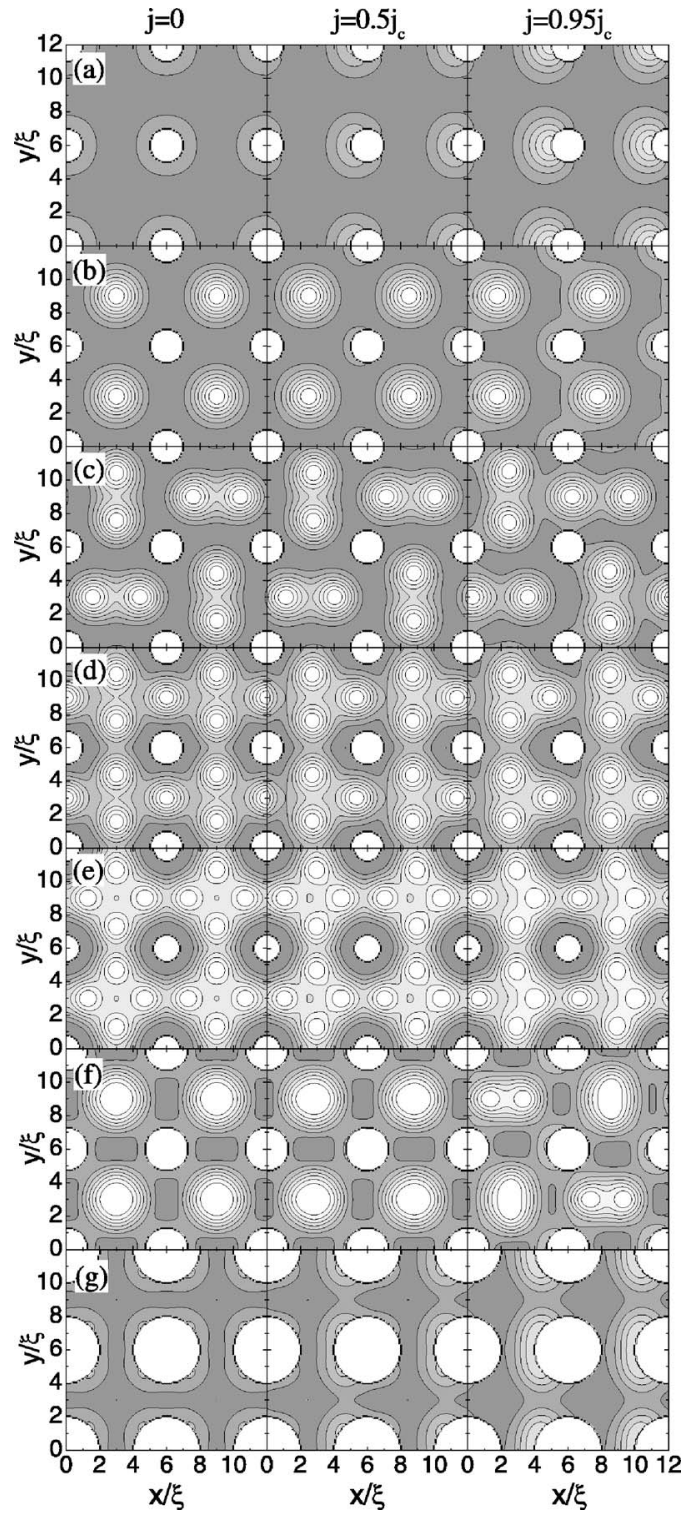

FIG. 16. Contour plots of the Cooper-pair density for $H=H_{1}$ (a), $H=H_{2}$ (b), $H=H_{3}$ (c,g), $H=H_{4}(\mathrm{~d}, \mathrm{f})$, and $H=H_{5}$ (e), and for the applied current $j=0$ (first column), $j=0.5 j_{c}$ (second column) and $j$ $=0.95 j_{c}$ (third column). GL parameter is $\kappa^{*}=10$, the period of the antidots lattice is $W=6 \xi$ and the radius of the antidots is $R=0.8 \xi(\mathrm{e})$, $R=1 \xi(\mathrm{a}-\mathrm{d}), R=1.3 \xi(\mathrm{f})$, and $R=2 \xi(\mathrm{g})$.

which is significantly higher than the thermal activation energy at this temperature $(k T=0.56 \mathrm{meV})$, but still low enough for successful switching by a relatively weak current. Moreover, when an ac is applied to the sample, the vortex can shift back and forth between the adjacent cells, resulting in resonant dissipation.

So far, we presented results at a fixed temperature. In what follows, we include temperature in our numerical analysis through the temperature dependence of the coherence length $\xi$. We now consider the superconducting film with thickness $d=20 \mathrm{~nm}$, interhole distance $W=1 \mu \mathrm{m}$, and antidot radius $R=0.2 \mu \mathrm{m}$. We choose the coherence length $\xi(0)=40 \mathrm{~nm}$ and the penetration depth $\lambda(0)=42 \mathrm{~nm}$, which are typical values for $\mathrm{Pb}$ films. Figure 17 shows the calcu-

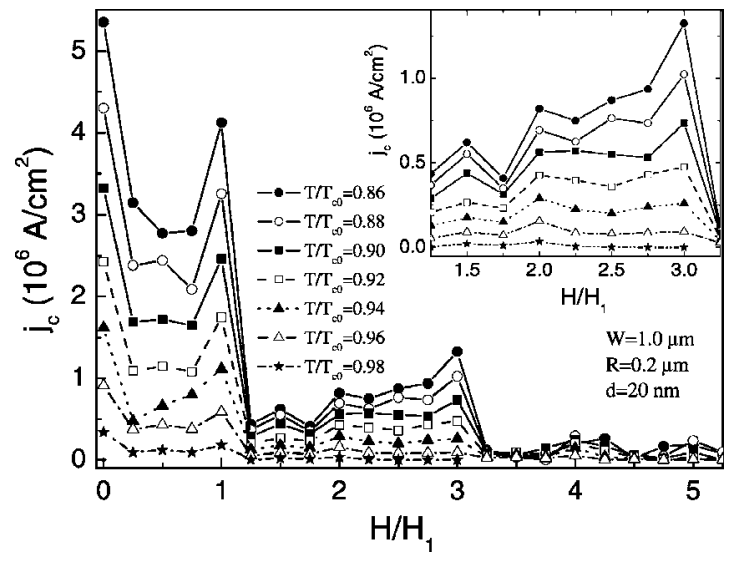

FIG. 17. Critical current density of the perforated superconducting film as a function of the applied magnetic field (in units of the first matching field $H_{1}$ ) at temperatures $T / T_{c 0}=0.86-0.98$. The lattice period is $W=1 \mu \mathrm{m}$, the antidot radius is $R=0.2 \mu \mathrm{m}$, and film thickness is $d=20 \mathrm{~nm}$.

lated critical current of the sample as a function of the applied field normalized to the first matching field at temperatures $T / T_{c 0}=0.86-0.98$. As expected, decreasing the temperature leads to a larger critical current for all values of the applied field. The relative height of the peak at zero field with respect to one at the first matching field increases with increasing temperature [see Fig. 18(a)]. At higher temperatures, i.e., for $\xi(T)>R$, a certain suppression of the order

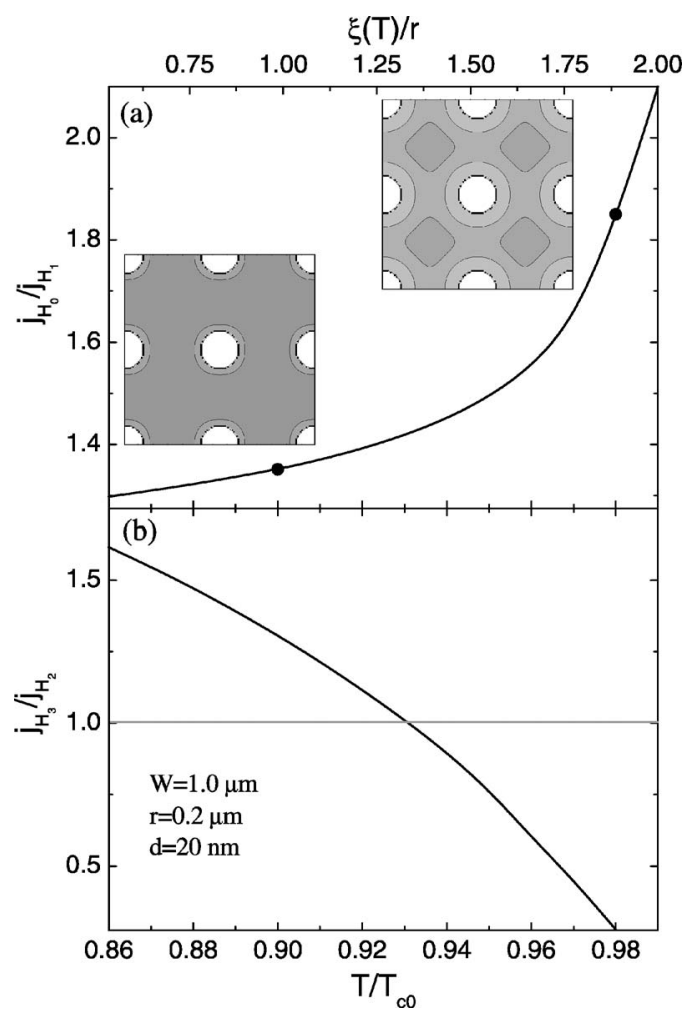

FIG. 18. The ratio of $j_{c}\left(H_{0}\right) / j_{c}\left(H_{1}\right)$ (a) and $j_{c}\left(H_{3}\right) / j_{c}\left(H_{2}\right)$ (b) as a function of temperature for the sample with parameters given in Fig. 17. The insets show the Cooper-pair density plots at temperatures indicated by the solid circles in the main figure (a). 


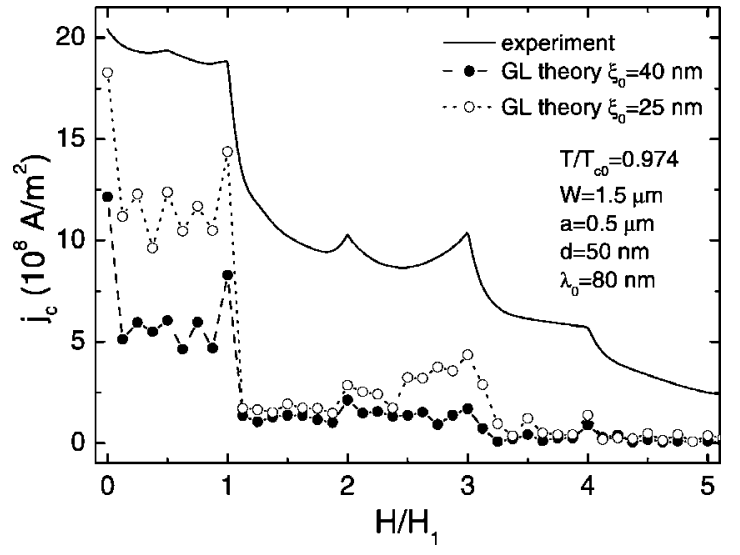

FIG. 19. Numerically obtained $j_{c}(H)$ characteristics of the superconducting film with antidot arrays (parameters given in the figure) for the coherence length at zero temperature $\xi(0)=40 \mathrm{~nm}$ (solid circles) and $\xi(0)=25 \mathrm{~nm}$ (open circles). The solid curve denotes experimental data (taken from Ref. 9).

parameter is present around the antidots [right-hand inset in Fig. 18(a)] as the core of pinned vortices overlaps with the interstitial regions. Consequently, the suppressed order parameter leads to a smaller $j_{c}$. The caging effect is found for temperatures $T \leqslant 0.93 T_{c 0}$ [see Fig. 18(b)] and it disappears with temperature when approaching $T_{c 0}$, since the vortices entirely cover the interstitial regions and effectively destroy superconductivity.

This effect that the critical current is larger for larger fields was recently observed experimentally. ${ }^{9}$ The considered sample was a $\mathrm{Pb}$ film of thickness $50 \mathrm{~nm}$, with square antidots of size $a=0.5 \mu \mathrm{m}$ and period $W=1.5 \mu \mathrm{m}$. The coherence length and the penetration depth at zero temperature were estimated to be $\xi(0)=40 \mathrm{~nm}$ and $\lambda(0)=80 \mathrm{~nm}$. Although plotted for other purposes, Fig. 6(b) in Ref. 9 demonstrates a clear overshoot of the critical current at $\mathrm{H}=\mathrm{H}_{3}$ with respect to the one at $H=H_{2}$, at the temperature $0.974 T_{c 0}$. Figure 19 shows the comparison of the calculated critical current density (dots) with experiment (solid line). Our $j_{c}(H)$ curve shows the same qualitative behavior as the experimental one, though a quantitative agreement is lacking for the experimentally estimated values of $\xi(0)$ and $\lambda(0)$. Better correspondence was achieved for smaller values of $\xi(0)$, indicating somewhat "dirty" sample in the experiment. No further attempts were made to improve the quantitative agreement with experiment because of the different determination of $j_{c}$ in the experiment and in our theory. In our calculations we use a dynamical criterium, i.e., we assume normal state as soon as vortices are set in motion, whereas in transport measurements a certain value of the threshold voltage was used to determine the critical current and the surface barrier at the edges is important. Therefore, our result should be considered as a lower limit to the experimental critical current. The qualitative behavior of $j_{c}$ at the matching fields should not be influenced by these facts.

\section{SUPERCONDUCTING-NORMAL $T_{c}(H)$ PHASE BOUNDARY}

The presence of antidot lattice in a superconducting film not only enhances the vortex pinning, which was discussed

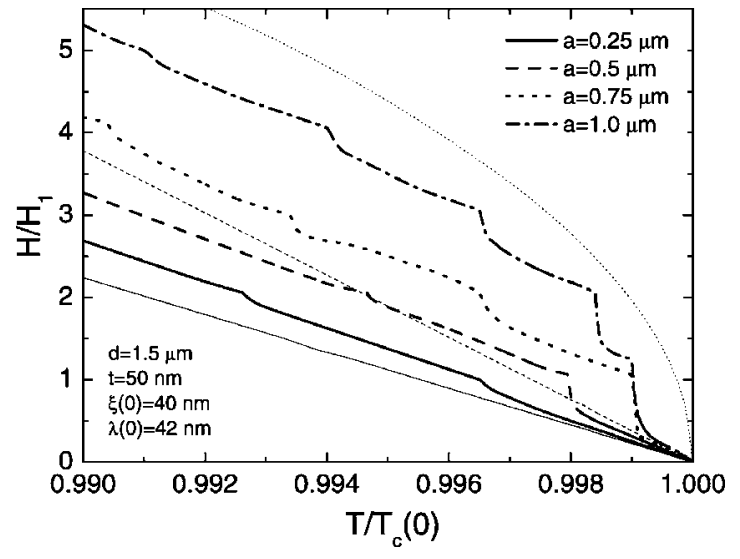

FIG. 20. $H-T$ phase boundary for the superconducting film with an antidot array. The film thickness is $d=50 \mathrm{~nm}$, the period of the antidot lattice is $W=1.5 \mu \mathrm{m}$, the antidot size is varied as $a$ $=0.25 \mu \mathrm{m}$ (solid curve), $a=0.5 \mu \mathrm{m}$ (dashed curve), $a=0.75 \mu \mathrm{m}$ (dotted curve), and $a=1.0 \mu \mathrm{m}$ (dashed-dotted curve). Thin solid curve denotes the upper critical field $\left(H_{c 2}\right)$ of the plain superconducting film [Eq. (5)], thin dashed curve gives the third critical field $H_{c 3}=1.69 H_{c 2}$ for a plain superconductor-vacuum boundary, and thin dotted curve is the critical field of a superconducting strip with thickness $\omega=0.5 \mu \mathrm{m}$ [Eq. (6)].

in the preceding section, but also affects substantially the nucleation of superconductivity. Due to the superconductingvacuum interface at the antidots, surface superconductivity will be important around each antidot, at fields above the bulk critical field $H_{c 2}(T)$. This makes it possible to enhance the critical field in patterned superconducting films above $H_{c 2}(T)$ and even beyond the third critical field $H_{c 3}(T)$. The ratio $H_{c 3}(T) / H_{c 2}(T)$ tends to the value 1.69 , the enhancement factor for a semi-infinite slab. ${ }^{38}$ However, for a dense antidot lattice a much larger enhancement can be achieved. Namely, if the antidots are sufficiently closely spaced, almost the entire sample may become superconducting at high fields through surface superconductivity.

The critical field of superconducting $\mathrm{Pb}$ films with a square array of antidots was investigated in Ref. 41 by the magnetoresistance measurements. The experimentally obtained $H-T$ phase boundary shows a cusplike behavior with cusps at integer and some fractional matching fields. The amplitude of the cusps depend on the resistive criterion: the cusps become sharper and their amplitude increases with decreasing this criterion.

We numerically investigated the $H-T$ phase boundary for a superconducting film of thickness $d=50 \mu \mathrm{m}$ in the presence of a regular array of square antidots with lattice period $W=1.5 \mu \mathrm{m}$. We take the coherence length at zero temperature as $\xi(0)=40 \mathrm{~nm}$ and penetration depth as $\lambda(0)=42 \mathrm{~nm}$. Figure 20 shows the calculated $T_{c}(H)$ phase diagram for different sizes of the antidots: $a=0.25 \mu \mathrm{m}$ (solid curve), $a$ $=0.5 \mu \mathrm{m}$ (dashed curve), $a=0.75 \mu \mathrm{m}$ (dotted curve), and $a$ $=1.0 \mu \mathrm{m}$ (dashed-dotted curve). For comparative reasons, we also plotted the phase boundary for a plain film (thin solid curve) with the same coherence length $\xi(0)=40 \mathrm{~nm}$, obtained from the well-known expression for the upper critical field, 


$$
H_{c 2}=\frac{\Phi_{0}}{2 \pi \xi^{2}(T)}=\frac{\Phi_{0}}{2 \pi \xi^{2}(0)}\left(1-T / T_{c 0}\right) .
$$

It can be easily seen that the antidot lattice has a profound influence on the critical magnetic field, as compared to a reference nonpatterned film. The critical temperature is enhanced at every field, and vice versa, regardless of the size of the antidots. Note also that matching features are present in $T_{c}(H)$ at integer matching fields. For small radius of the antidots matching peaks at higher integer matching fields $H$ $>\mathrm{H}_{2}$ are weakly pronounced, due to the small holesaturation number (see Sec. III). We did not observe clear evidence of fractional matching features.

For small radius of the antidots the sample basically acts as a nonpatterned film for temperatures close to $T_{c 0}$ and the dependence of the critical temperature on the applied field is almost linear. For larger sizes of the antidots (e.g., dotted and dashed-dotted curves in Fig. 20), the critical field becomes substantially higher than the third critical field of a semiinfinite slab (thin dashed curve), and the peaks at matching fields are more pronounced. In addition, $T_{c}(H)$ exhibits a parabolic background as for a thin slab in a perpendicular field, as well as to a thin film in a parallel field, which can be described in the London limit by ${ }^{36}$

$$
H_{c 3}=\frac{\sqrt{12} \xi(T)}{\omega} H_{c 2}(T),
$$

where $\omega$ stands for the width of the superconducting strip.

\section{CONCLUSIONS}

We have studied the vortex structure of a thin superconducting film with a regular array of antidots, which shows a rich variety of ordered vortex lattice configurations for different matching and fractional matching fields $H_{n}$. For small radius of the holes, the vortex configurations with one vortex captured in each hole and the others located in the interstitial sites are realized, where interstitial vortices form regular patterns, either as multivortices or giant vortices, or combination of giant vortex and multivortex states. For particular geometrical parameters of the sample and the applied field, a symmetry imposed vortex-antivortex configuration is found. Depending on the ratio between the hole radius $R$ and the interhole distance $W$, multiquanta vortices may be forced into the antidots, in spite of their low saturation number at smaller magnetic fields. To illustrate the transition between possible multiquanta states in the holes we showed a diagram of the occupation number $n_{o}$ as a function of the radius of the holes and interhole distance for different values of the effective GL parameter. $n_{o}$ increases with decreasing $\kappa^{*}$ due to the enhanced expulsion of the magnetic field from the superconductor and giant vortices become energetically favorable because of the attractive interaction between the vortices.

When the pinning force of the antidots is small, i.e., small radius of the antidots, the triangular vortex lattice becomes energetically favorable. Depending on the applied field all the vortices can be located between the antidots, or some of them are pinned by the antidots and some of them are located between the pinning centers. We calculated the phase diagram which shows the transition between the triangular and pinned square vortex lattices for two values of the applied field and GL parameter $\kappa$. We found that the results from the simple London theory for the phase diagram are different from our GL results for different applied fields. Moreover, we could not find triangular vortex structures for the fields $H=H_{1}$ and $H=H_{1 / 2}$ as a ground state for a dense antidot lattice.

The critical current $j_{c}$ of the sample shows well-defined peaks at different matching $H_{n}$ and fractional matching fields, indicating that vortices are strongly pinned by antidots. However, the level of $j_{c}$ enhancement at particular magnetic field strongly depends on the antidot occupation number $n_{o}$. For certain parameters of the sample, the critical current becomes larger at higher matching fields, contrary to conventional behavior.

We also studied the $T_{c}(H)$ phase boundary of regularly perforated superconducting film. When an antidot array is present the critical temperature $T_{c}(H)$ is enhanced compared to a nonpatterned film and distinct cusps in the phase boundary are found for different matching fields, which is in agreement with experiment. ${ }^{41}$ This behavior is in contrast to the Little-Parks ${ }^{42}$ type structures found in finite size superconductors. ${ }^{43}$ The increase of the antidot size for given lattice period leads to the change of the $T_{c}(H)$ background from linear to parabolic behavior except for $T$ near $T_{c 0}$.

\section{ACKNOWLEDGMENTS}

This work was supported by the Flemish Science Foundation (FWO-Vl), the Belgian Science Policy (IUAP), the JSPS-ESF NES program, and the ESF-AQDJJ network.
*Electronic address: golibjon.berdiyorov@ua.ac.be

†Electronic address: francois.peeters@ua.ac.be

${ }^{1}$ K. Harada, O. Kamimura, H. Kasai, T. Matsuda, A. Tonomura, and V. V Moshchalkov, Science 274, 1167 (1996).

${ }^{2}$ M. Baert, V. V. Metlushko, R. Jonckheere, V. V. Moshchalkov, and Y. Bruynseraede, Phys. Rev. Lett. 74, 3269 (1995).

${ }^{3}$ V. V. Moshchalkov, M. Baert, V. V. Metlushko, E. Rosseel, M. J. Van Bael, K. Temst, R. Jonckheere, and Y. Bruynseraede, Phys. Rev. B 54, 7385 (1996).
${ }^{4}$ V. V. Moshchalkov, M. Baert, V. V. Metlushko, E. Rosseel, M. J. Van Bael, K. Temst, Y. Bruynseraede, and R. Jonckheere, Phys. Rev. B 57, 3615 (1998).

${ }^{5}$ V. Metlushko, U. Welp, G. W. Crabtree, R. Osgood, S. D. Bader, L. E. DeLong, Zhao Zhang, S. R. J. Brueck, B. Ilic, K. Chung, and P. J. Hesketh, Phys. Rev. B 60, R12585 (1999); V. V. Metlushko, L. E. DeLong, M. Baert, E. Rosseel, M. J. Van Bael, K. Temst, V. V. Moshchalkov, and Y. Bruynseraede, Europhys. Lett. 41, 333 (1998). 
${ }^{6}$ L. Van Look, B. Y. Zhu, R. Jonckheere, B. R. Zhao, Z. X. Zhao, and V. V. Moshchalkov, Phys. Rev. B 66, 214511 (2002).

${ }^{7}$ A. V. Silhanek, S. Raedts, M. Lange, and V. V. Moshchalkov, Phys. Rev. B 67, 064502 (2003).

${ }^{8}$ A. V. Silhanek, S. Raedts, M. J. Van Bael, and V. V. Moshchalkov, Phys. Rev. B 70, 054515 (2004).

${ }^{9}$ A. V. Silhanek, L. Van Look, R. Jonckheere, B. Y. Zhu, S. Raedts, and V. V. Moshchalkov, Phys. Rev. B 72, 014507 (2005).

${ }^{10}$ S. Raedts, A. V. Silhanek, M. J. Van Bael, and V. V. Moshchalkov, Phys. Rev. B 70, 024509 (2004).

${ }^{11}$ A. N. Grigorenko, G. D. Howells, S. J. Bending, J. Bekaert, M. J. Van Bael, L. Van Look, V. V. Moshchalkov, Y. Bruynseraede, G. Borghs, I. I. Kaya, and R. A. Stradling, Phys. Rev. B 63, 052504 (2001).

${ }^{12}$ M. M. Doria and G. F. Zebende, Phys. Rev. B 66, 064519 (2002).

${ }^{13}$ J. I. Martin, M. Velez, J. Nogues, and Ivan K. Schuller, Phys. Rev. Lett. 79, 1929 (1997).

${ }^{14}$ J. I. Martin, M. Velez, A. Hoffmann, I. K. Schuller, and J. L. Vicent, Phys. Rev. Lett. 83, 1022 (1999).

${ }^{15}$ M. V. Milošević and F. M. Peeters, Phys. Rev. Lett. 93, 267006 (2004).

${ }^{16}$ A. A. Abrikosov, Zh. Eksp. Teor. Fiz. 32, 1442 (1957).

${ }^{17}$ W. H. Kleiner, L. M. Roth, and S. H. Autler, Phys. Rev. 133, A1226 (1964).

${ }^{18}$ C. Reichhardt, J. Groth, C. J. Olson, Stuart B. Field, and Franco Nori, Phys. Rev. B 54, 16108 (1997).

${ }^{19}$ C. Reichhardt, C. J. Olson, and Franco Nori, Phys. Rev. B 57, 7937 (1998).

${ }^{20}$ C. Reichhardt, G. T. Zimanyi, and N. Gronbech-Jensen, Phys. Rev. B 64, 014501 (2001).

${ }^{21}$ C. Reichhardt and N. Gronbech-Jensen, Phys. Rev. B 63, 054510 (2001).

${ }^{22}$ B. Y. Zhu, F. Marchesoni, V. V. Moshchalkov, and F. Nori, Phys. Rev. B 68, 014514 (2003).

${ }^{23}$ G. Karapetrov, J. Fedor, M. Iavarone, D. Rosenmann, and W. K. Kwok, Phys. Rev. Lett. 95, 167002 (2005).
${ }^{24}$ H. Nordborg and V. M. Vinokur, Phys. Rev. B 62, 12408 (2000).

${ }^{25}$ G. S. Mkrtchyan and V. V. Shmidt, Sov. Phys. JETP 34, 195 (1972).

${ }^{26}$ M. Doria, S. C. de Andrade, and E. Sardella, Physica C 341-348, 1199 (2000).

${ }^{27}$ A. Bezryadin and B. Pannetier, Phys. Scr. 66, 225 (1996).

${ }^{28}$ A. I. Buzdin, Phys. Rev. B 47, 11416 (1993).

${ }^{29}$ A. Bezryadin and B. Pannetier, J. Low Temp. Phys. 98, 251 (1995).

${ }^{30}$ V. A. Schweigert, F. M. Peeters, and P. S. Deo, Phys. Rev. Lett. 81, 2783 (1998).

${ }^{31}$ V. A. Schweigert and F. M. Peeters, Phys. Rev. B 57, 13817 (1998).

${ }^{32}$ M. M. Doria, J. E. Gubernatis, and D. Rainer, Phys. Rev. B 39, 9573 (1989).

${ }^{33}$ R. Kato, Y. Enomoto, and S. Maekawa, Phys. Rev. B 47, 8016 (1993).

${ }^{34}$ G. R. Berdiyorov, M. V. Milošević, and F. M. Peeters, Phys. Rev. Lett. 96, 207001 (2006).

${ }^{35}$ G. R. Berdiyorov, M. V. Milošević, and F. M. Peeters, Physica C 437-438, 25 (2006).

${ }^{36}$ M. Tinkham, Introduction to Superconductivity (McGraw-Hill, New York, 1996).

${ }^{37}$ G. Stenuit, S. Michotte, J. Govaerts, and L. Piraux, Semicond. Sci. Technol. 18, 174 (2005).

${ }^{38}$ P. G. de Gennes, Superconductivity of Metals and Alloys (Benjamin, New York, 1966).

${ }^{39}$ W. V. Pogosov, A. L. Rakhmanov, and V. V. Moshchalkov, Phys. Rev. B 67, 014532 (2003).

${ }^{40}$ G. R. Berdiyorov, M. V. Milošević, and F. M. Peeters, Europhys. Lett. 74, 493 (2006).

${ }^{41}$ E. Rosseel, T. Puig, M. Baert, M. J. Van Bael, V. V. Moshchalkov, and Y. Bruynseraede, Physica C 282-287, 1567 (1997).

${ }^{42}$ W. A. Little and R. Parks, Phys. Rev. 113, A97 (1964).

${ }^{43}$ G. R. Berdiyorov, B. J. Baelus, M. V. Milošević, and F. M. Peeters, Phys. Rev. B 68, 174521 (2003). 